TITLE:

\title{
Multi-agent consensus under a communication-broadcast mixed environment
}

$\operatorname{AUTHOR}(S)$ :

Azuma, Shun-ichi; Tanaka, Yosuke; Sugie, Toshiharu

\section{CITATION:}

Azuma, Shun-ichi ...[et al]. Multi-agent consensus under a communication-broadcast mixed environment. International Journal of Control 2014, 87(6): 1103-1116

ISSUE DATE:

2014-06-03

URL:

http://hdl.handle.net/2433/187106

\section{RIGHT:}

(c) 2014 The Author(s). Published by Taylor \& Francis. This is an Open Access article. Non-commercial re-use, distribution, and reproduction in any medium, provided the original work is properly attributed, cited, and is not altered, transformed, or built upon in any way, is permitted. The moral rights of the named author(s) have been asserted. Permission is granted subject to the terms of the License under which the work was published. Please check the License conditions for the work which you wish to reuse. Full and appropriate attribution must be given. This permission does not cover any third party copyrighted material which may appear in the work requested. 


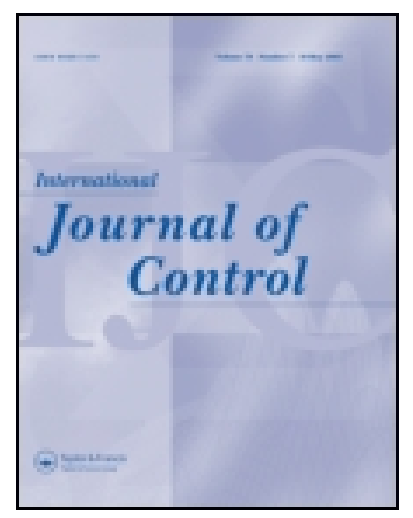

\title{
International J ournal of Control
}

Publication details, including instructions for authors and subscription information: http:// www. tandfonline.com/loi/tcon20

\section{Multi-agent consensus under a communication-broadcast mixed environment}

\author{
Shun-ichi Azuma ${ }^{a}$, Yosuke Tanaka ${ }^{a} \&$ Toshiharu Sugie ${ }^{a}$ \\ ${ }^{a}$ Graduate School of Informatics, Kyoto University, Uji, Kyoto 611-0011, Japan \\ Accepted author version posted online: 26 Nov 2013. Published online: 05 Feb 2014.
}

To cite this article: Shun-ichi Azuma, Yosuke Tanaka \& Toshiharu Sugie (2014) Multi-agent consensus under a communication-broadcast mixed environment, International J ournal of Control, 87:6, 1103-1116, DOI: $10.1080 / 00207179.2013 .868608$

To link to this article: http:// dx. doi.org/ 10.1080/00207179.2013.868608

\section{PLEASE SCROLL DOWN FOR ARTICLE}

Taylor \& Francis makes every effort to ensure the accuracy of all the information (the "Content") contained in the publications on our platform. Taylor \& Francis, our agents, and our licensors make no representations or warranties whatsoever as to the accuracy, completeness, or suitability for any purpose of the Content. Versions of published Taylor \& Francis and Routledge Open articles and Taylor \& Francis and Routledge Open Select articles posted to institutional or subject repositories or any other third-party website are without warranty from Taylor \& Francis of any kind, either expressed or implied, including, but not limited to, warranties of merchantability, fitness for a particular purpose, or non-infringement. Any opinions and views expressed in this article are the opinions and views of the authors, and are not the views of or endorsed by Taylor \& Francis. The accuracy of the Content should not be relied upon and should be independently verified with primary sources of information. Taylor \& Francis shall not be liable for any losses, actions, claims, proceedings, demands, costs, expenses, damages, and other liabilities whatsoever or howsoever caused arising directly or indirectly in connection with, in relation to or arising out of the use of the Content.

This article may be used for research, teaching, and private study purposes. Terms \& Conditions of access and use can be found at http://www.tandfonline.com/page/terms-and-conditions

It is essential that you check the license status of any given Open and Open Select article to confirm conditions of access and use. 


\title{
Multi-agent consensus under a communication-broadcast mixed environment
}

\author{
Shun-ichi Azuma*, Yosuke Tanaka and Toshiharu Sugie \\ Graduate School of Informatics, Kyoto University, Uji, Kyoto 611-0011, Japan
}

(Received 23 March 2013; accepted 19 November 2013)

\begin{abstract}
This paper addresses multi-agent control under an environment where both agent-to-agent communication and one-to-all broadcast are available. The problem studied here is a consensus problem for a pre-specified location, i.e. collecting the agents at a desired location in an $n$-dimensional space, with a limited communication range and an unlimited broadcast range, which is a nice example to be solved under the mixed environment. For solving this, we first introduce a concept of the connected agent group. Using this notion, the problem is reduced to a consensus problem for the group-to-group relation and that for the agent-to-agent relation in the groups, from which we derive a controller achieving the consensus with probability 1. Finally, the performance of the proposed controller is demonstrated by numerical simulation.
\end{abstract}

Keywords: multi-agent system; broadcast control; consensus problem

\section{Introduction}

Multi-agent control has received much attention in the control community. The reason lies in its great potential for socially beneficial applications, such as smart buildings, smart transportation, and smart grids. In fact, as easily imagined, one of the key techniques to realise such applications is the control of systems involving numerous components.

The problem setting mainly considered in this community is summarised as follows. Suppose that a number of agents are given and assume that the agent-to-agent communication is available as shown in Figure 1. The problems of interest are (i) to analyse the agent actions and the network topology and (ii) to design them (or some of them) for achieving a given global objective. A number of results have been obtained for these types of problems (see, e.g. Abdallah \& Tanner, 2007; Martinez, Cortés, \& Bullo, 2007; Mesbahi \& Egerstedt, 2010; Olfati-Saber, Fax, \& Murray, 2007; Sundaram \& Hadjicostis, 2008, and references therein). Meanwhile, a different setting, which is illustrated in Figure 2, has been proposed most recently (Bretl, 2007; Ueda, Odhner, \& Asada, 2007). It is assumed there that the one-to-all broadcast is available instead of the agent-to-agent communication. Namely, the system is assumed to be controlled by sending the same signal to all the agents in an indiscriminate manner. Under this setting, analysis and design problems with respect to the agent actions and/or the broadcast signal have been considered (Azuma, Baba, \& Sugie, 2012; Azuma, Yoshimura, \& Sugie, 2013; Bretl, 2007; Das \& Ghose, 2009; Julius et al., 2008; Ueda et al., 2007; Wood, Das, \& Asada, 2008).
These two settings are addressed from different standpoints, and so they have been studied independently. On the other hand, there are cases where both the agent-to-agent communication and the one-to-all broadcast are available at the same time (or can be set to available). However, the mixed setting has never been handled so far, although the synergy could be expected.

This paper thus addresses a multi-agent problem under a mixed environment of communication and broadcast, as shown in Figure 3. In particular, we consider here a consensus problem for a pre-specified location, i.e. collecting the agents at a desired location in an $n$-dimensional space, with a limited communication range and an unlimited broadcast range. In the case of Figure 1 where only the communication is available, the consensus can be accomplished only if all the agents are connected in terms of information flow (Olfati-Saber et al., 2007). That is, the problem cannot be solved if the agents are initially placed far away from each other. On the other hand, the problem can be solved even if only the broadcast is available, as shown in Figure 2, while it is known that it takes an impractically long time (Azuma et al., 2013). So a practical solution cannot be obtained only with the broadcast. Under the above circumstances, the problem is a nice example to be solved under the mixed environment.

In this paper, we solve the problem based on the decomposition into global consensus and local consensus. To this end, we introduce a concept of the connected agent groups. Using this notion, the problem is reduced into a consensus problem for the group-to-group relation and that for the

\footnotetext{
${ }^{*}$ Corresponding author. Email: sazuma@i.kyoto-u.ac.jp
} 


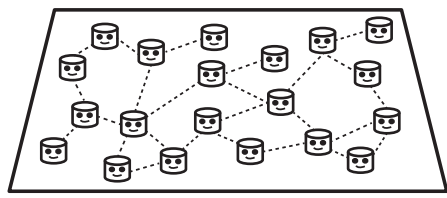

Figure 1. Multi-agent control with communication.
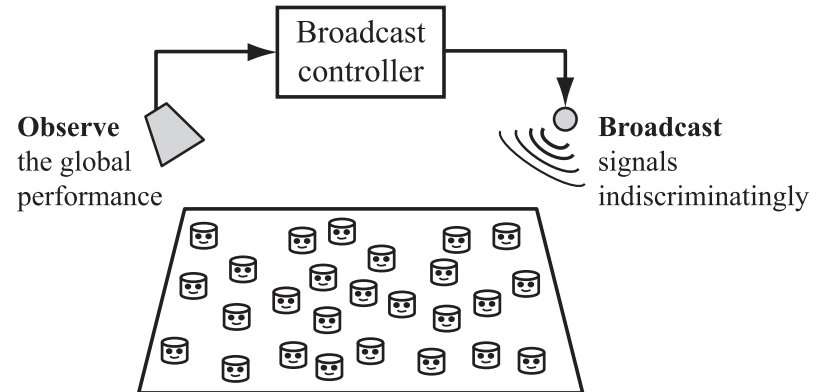

Figure 2. Multi-agent control with broadcast.

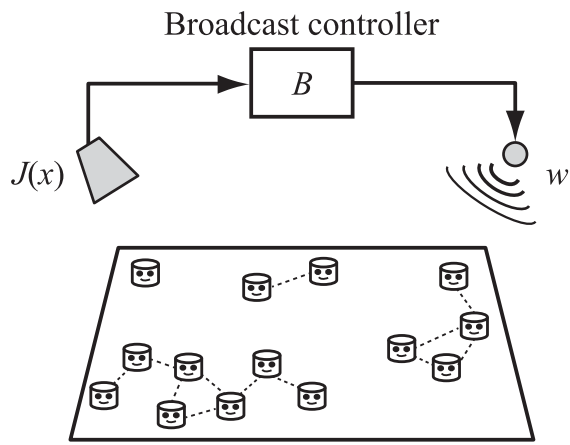

Figure 3. Feedback system $\Sigma$ under a broadcast-communication mixed environment.

agent-to-agent relation in the groups. Our solution is then presented as a combination of a group-to-group consensus controller and an agent-to-agent consensus controller. The subcontrollers fully utilise the broadcast and the communication, respectively. The proposed controller is proved to achieve the consensus with probability 1 (w.p.1). Finally, the performance is demonstrated by numerical simulation.

This paper is based on our earlier preliminary version (Azuma et al., 2012), published in a conference proceedings, and contains full explanations, proofs, and extensions omitted there.

Notation: Let $\mathbf{R}, \mathbf{R}_{+}$, and $\mathbf{R}_{0+}$ be the real number field, the set of positive real numbers, and the set of non-negative real numbers, respectively. We denote by $0, \mathbf{1}_{n}$, and $I_{n}$ the zero scalar/vector, the $n \times 1$ vector whose elements are 1 , and the $n \times n$ identity matrix. The signum function used here is defined as

$$
\operatorname{sign}(a):=\left\{\begin{aligned}
1 & \text { if } 0 \leq a \\
-1 & \text { if } a<0
\end{aligned}\right.
$$

where $a \in \mathbf{R}$. For the vector $x$, let $\|x\|$ and $\operatorname{sign}(x)$ be the Euclidian norm and the vector obtained by applying the above signum function elementwise. For the vector $x$ with non-zero elements, we use $x^{(-1)}$ to represent the elementwise inverse, e.g. $x^{(-1)}=\left[\begin{array}{ll}1 / 4 & 1 / 3\end{array}\right]^{\top}$ for $x=\left[\begin{array}{ll}4 & 3\end{array}\right]^{\top}$. The gradient of the differentiable function $J: \mathbf{R}^{n} \rightarrow \mathbf{R}$ is expressed by $\nabla J(x)$ (note $\nabla J(x) \in \mathbf{R}^{n}$ ), and the Kronecker product for the matrices $A$ and $B$ is represented by $A \otimes B$. Let $\mathbb{E}(a \mid b)$ be the conditional expectation for the random variables $a$ and $b$. For the stochastic process $z_{0}, z_{1}, \ldots$, if the probability that $\lim _{t \rightarrow \infty} z_{t}=z_{*}$ is 1 , then we say that the stochastic process converges to $z_{*}$ almost surely. This type of convergence is called the almost-sure convergence and expressed as " $\lim _{t \rightarrow \infty} z_{t}=z_{*}$ w.p.1". We denote by $|\mathbf{S}|$, $\min (\mathbf{S})$, and $\mathbf{P}(\mathbf{S})$ the cardinality, the minimum element, and the power set of the set $\mathbf{S}$. For the sets $\mathbf{S}_{1}$ and $\mathbf{S}_{2}$, the relation $\mathbf{S}_{1} \subset \mathbf{S}_{2}\left(\mathbf{S}_{1} \subseteq \mathbf{S}_{2}\right)$ means that $\mathbf{S}_{1}$ is a strict subset (a subset) of $\mathbf{S}_{2}$, and $\mathbf{S}_{1} \oplus \mathbf{S}_{2}$ represents the Minkowski sum, i.e. $\mathbf{S}_{1} \oplus \mathbf{S}_{2}=\left\{x_{1}+x_{2} \mid x_{1} \in \mathbf{S}_{1}, x_{2} \in \mathbf{S}_{2}\right\}$. For the multiset $\mathbf{M}$ of scalars (whose elements are allowed to appear more than once), let abmax(M) be the maximum element in absolute value. If there are multiple maximum elements in this sense, $\operatorname{abmax}(\mathbf{M})$ takes the maximum positive element. For example, abmax $(\mathbf{M})=-6$ for $\mathbf{M}=\{-6,5,1,2\}$ and $\operatorname{abmax}(\mathbf{M})=6$ for $\mathbf{M}=\{-6,6,1,2\}$. The function is similarly defined for the multi-set of column vectors. More precisely, for $\mathbf{M}=\left\{x_{1}, x_{2}, \ldots, x_{m}\right\}\left(x_{i} \in \mathbf{R}^{n}\right)$,

$$
\operatorname{abmax}(\mathbf{M}):=\left[\begin{array}{c}
\operatorname{abmax}\left(\left\{x_{11}, x_{21}, \ldots, x_{m 1}\right\}\right) \\
\operatorname{abmax}\left(\left\{x_{12}, x_{22}, \ldots, x_{m 2}\right\}\right) \\
\vdots \\
\operatorname{abmax}\left(\left\{x_{1 n}, x_{2 n}, \ldots, x_{m n}\right\}\right)
\end{array}\right]
$$

where $x_{i j} \in \mathbf{R}$ is the $j$ th element of $x_{i}$. For the vectors $x_{1}$, $x_{2}, \ldots \in \mathbf{R}^{n}$ and the set $\mathbf{I}:=\left\{i_{1}, i_{2}, \ldots, i_{m}\right\} \subset\{1,2, \ldots\}$, let $\left\{x_{i}\right\}_{i \in \mathbf{I}}:=\left\{x_{i_{1}}, x_{i_{2}}, \ldots, x_{i_{m}}\right\} \in \mathbf{P}\left(\mathbf{R}^{n}\right)$. Similar notations are often used, e.g. $\left\{x_{i}-y\right\}_{i \in \mathbf{I}}$ for $\left\{x_{i_{1}}-y, x_{i_{2}}-y, \ldots\right.$, $\left.x_{i_{m}}-y\right\}$. Finally, consider the finite set $\mathbf{A}:=\left\{a_{1}, a_{2}, \ldots\right.$, $\left.a_{m}\right\} \subset \mathbf{R}$. Let $\lfloor\theta\rfloor_{\mathbf{A}}$ be the maximum element of $\mathbf{A}$ not more than the number $\theta \in \mathbf{R}$, and let $\operatorname{vec}_{n}(\mathbf{A})$ be the vector $a:=\left[\begin{array}{llllllll}\tilde{a}_{1} & \tilde{a}_{2} & \cdots & \tilde{a}_{m} & 0 & 0 & \cdots & 0\end{array}\right]^{\top} \in \mathbf{R}^{n}$ such that $\left\{\tilde{a}_{1}, \tilde{a}_{2}, \ldots, \tilde{a}_{m}\right\}=\mathbf{A}$ for $n \in\{m, m+1, \ldots\}$. Conversely,

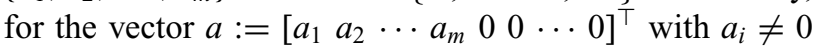
$(i=1,2, \ldots, m)$, we use $\mathbf{S}(a)$ to express the set $\left\{a_{1}, a_{2}, \ldots\right.$, $\left.a_{m}\right\}$. Note here that $\mathbf{S}\left(\operatorname{vec}_{n}(\mathbf{A})\right)=\mathbf{A}$ if $a_{i} \neq 0(i=1,2, \ldots$, $m)$.

\section{Problem formulation}

\subsection{System description}

Consider the feedback system $\Sigma$ in Figure 3, composed of $N$ agents and a broadcast controller.

Agent $i$ is a mobile robot embedding a local controller, as shown in Figure 4. The physical dynamics is described 


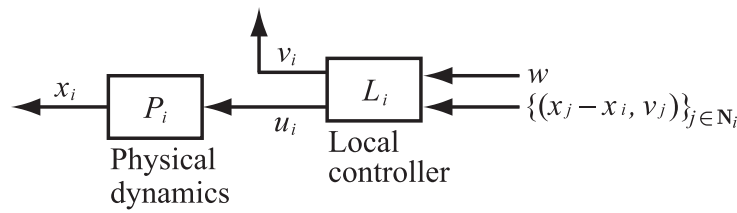

Figure 4. Block diagram of agent $i$.

by

$$
P_{i}: x_{i}(t+1)=x_{i}(t)+u_{i}(t)
$$

where $x_{i}(t) \in \mathbf{R}^{n}$ is the position, $u_{i}(t) \in \mathbf{R}^{n}$ is the control input, and $t \in\{0,1, \ldots\}$ is the discrete time. The embedded local controller is of the form

$L_{i}:\left\{\begin{array}{l}\xi_{i}(t+1)=\alpha\left(\xi_{i}(t),\left\{\left(x_{j}(t)-x_{i}(t), v_{j}(t)\right)\right\}_{j \in \mathbf{N}_{i}(t)}, w(t)\right) \\ u_{i}(t)=\beta_{1}\left(\xi_{i}(t),\left\{\left(x_{j}(t)-x_{i}(t), v_{j}(t)\right)\right\}_{j \in \mathbf{N}_{i}(t)}, w(t)\right) \\ v_{i}(t)=\beta_{2}\left(\xi_{i}(t),\left\{\left(x_{j}(t)-x_{i}(t), v_{j}(t)\right)\right\}_{j \in \mathbf{N}_{i}(t)}, w(t)\right)\end{array}\right.$

where $\xi_{i}(t) \in \mathbf{R}^{v}$ is the state (corresponding to a memory), $\left\{\left(x_{j}(t)-x_{i}(t), v_{j}(t)\right)\right\}_{j \in \mathbf{N}_{i}(t)} \in \mathbf{P}\left(\mathbf{R}^{n} \times \mathbf{R}^{m}\right)$ and $w(t) \in \mathbf{R}$ are the inputs, $u_{i}(t)$ and $v_{i}(t) \in \mathbf{R}^{m}$ are the outputs, and $\alpha: \mathbf{R}^{v} \times \mathbf{P}\left(\mathbf{R}^{n} \times \mathbf{R}^{m}\right) \times \mathbf{R} \rightarrow \mathbf{R}^{v}, \beta_{1}: \mathbf{R}^{v} \times \mathbf{P}\left(\mathbf{R}^{n}\right.$ $\left.\times \mathbf{R}^{m}\right) \times \mathbf{R} \rightarrow \mathbf{R}^{n}$, and $\beta_{2}: \mathbf{R}^{v} \times \mathbf{P}\left(\mathbf{R}^{n} \times \mathbf{R}^{m}\right) \times$ $\mathbf{R} \rightarrow \mathbf{R}^{m}$ are functions. The set $\mathbf{N}_{i}(t) \subseteq\{1,2, \ldots, N\}$ is the index set of the neighbours, i.e. the agents whose information is available to agent $i$. The functions $\alpha, \beta_{1}$, and $\beta_{2}$ are assumed to be the same for all the local controllers $L_{i}$ $(i=1,2, \ldots, N)$, which means that the agents are handled indiscriminately. by

On the other hand, the broadcast controller $B$ is given

$$
B: w(t)=\gamma(J(x(t)))
$$

where $J(x(t)) \in \mathbf{R}_{0+}$ is the input, $w(t) \in \mathbf{R}$ is the output, which is called the broadcast signal, and $\gamma: \mathbf{R}_{0+} \rightarrow \mathbf{R}$ is a function. The vector $x(t) \in \mathbf{R}^{n N}$ is the collective position of the agents, i.e. $x(t):=\left[x_{1}^{\top}(t) x_{2}^{\top}(t) \cdots x_{N}^{\top}(t)\right]^{\top}$, and $J$ : $\mathbf{R}^{n N} \rightarrow \mathbf{R}_{0+}$ is the performance index which quantifies the global performance $x(t)$ of the system $\Sigma$.

In the system $\Sigma$, the agents move in the $n$-dimensional space according to both the local information through the communication with the neighbours (or the sensing of the neighbours) and the global information through the broadcast. Then, the broadcast controller $B$ observes the global performance $J(x(t))$ and broadcasts the signal $w(t)$ to govern the global behaviour. The local controllers $L_{i}(i=$ $1,2, \ldots, N)$, on the other hand, determine the local actions of the agents with the relative positions to the neighbours (i.e. $\left.\left\{x_{j}(t)-x_{i}(t)\right\}_{j \in \mathbf{N}_{i}(t)}\right)$, the information sent by the neighbours (i.e. $\left\{v_{j}(t)\right\}_{j \in \mathbf{N}_{i}(t)}$ ), and the broadcast signal $w(t)$. Note here that agent $i$ can know the relative positions $\left\{x_{j}(t)-x_{i}(t)\right\}_{j \in \mathbf{N}_{i}(t)}$ without knowing its own position $x_{i}(t)$ in the world coordinate frame, because the difference between $x_{j}(t)$ and $x_{i}(t)$ can be directly measured in the body fixed frame of agent $i$.

\subsection{Consensus problem under a broadcast-communication mixed environment}

In this paper, we consider the following neighbour set:

$$
\begin{aligned}
& \mathbf{N}_{i}(t):=\{j \in\{1,2, \ldots, N\} \backslash\{i\} \mid \\
&\left.\exists s \in[0, t] \text { s.t. }\left\|x_{j}(s)-x_{i}(s)\right\|<r\right\}
\end{aligned}
$$

where $r \in \mathbf{R}_{+}$is a given constant. Agent $j \in \mathbf{N}_{i}(t)$ is the agent that has entered in the $r$-ball of agent $i$ before time $t$. The constant $r$ then corresponds to the distance to establish the communication. By definition, the elements of $\mathbf{N}_{i}(t)$ is non-decreasing with respect to $t$, i.e.

$$
\mathbf{N}_{i}(t) \subseteq \mathbf{N}_{i}(t+1)
$$

for every $i \in\{1,2, \ldots, N\}$ and $t \in\{0,1, \ldots\}$.

We address here the consensus problem for a prespecified location, that is, the problem of collecting the agents at the desired location $x_{d} \in \mathbf{R}^{n}$. The achievement of the task can be quantified by

$$
J(x(t)):=\sum_{i=1}^{N}\left\|x_{i}(t)-x_{d}\right\|^{2}
$$

for which $J(x(\infty))=0$ implies that the consensus $x_{i}(\infty)$ $=x_{d}(i=1,2, \ldots, N)$ is completed. Then, our problem is formulated as follows.

Problem 1: For the system $\Sigma$ in Figure 3, the distance $r \in$ $\mathbf{R}_{+}$and the desired location $x_{d} \in \mathbf{R}^{n}$ are given. Find local controllers $L_{i}(i=1,2, \ldots, N)$ and a broadcast controller $B$ (i.e. find functions $\alpha, \beta$, and $\gamma$ ) such that

$$
\lim _{t \rightarrow \infty} J(x(t))=0
$$

for every initial state $x(0) \in \mathbf{R}^{n N}$ and $\left(\xi_{1}(0), \xi_{2}(0), \ldots\right.$, $\left.\xi_{N}(0)\right) \in \mathbf{R}^{v N}$.

Several remarks on the problem are given.

First, there is no trivial solution though the consensus point is pre-specified. In fact, as seen in (2), the agents have the information not on their positions in the world coordinate frame, but on the relative positions $\left\{x_{j}(t)-x_{i}(t)\right\}_{j \in \mathbf{N}_{i}(t)}$ to the neighbours, and so they do not know where the desired location $x_{d}$ is. Meanwhile, the agents can obtain the global information through the broadcast signal containing the information of $J(x(t))$; however, 


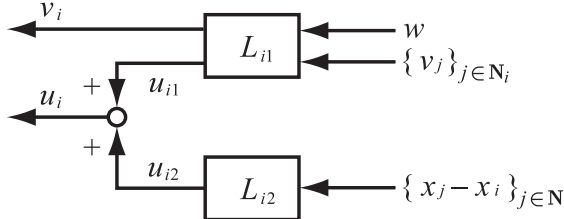

Figure 5. Structure of the local controller $L_{i}$.

$J(x(t))$ is highly compressed information on the agents' positions. In this sense, Problem 1 is challenging.

Second, the neighbour set defined above is different from the so-called $r$-disk proximity neighbour set, i.e. $\left\{j \in\{1,2, \ldots, N\} \backslash\{i\} \mid\left\|x_{i}(t)-x_{j}(t)\right\|<r\right\}$, which may fit more realistic situations. However, the following results can be straightforwardly extended to the case with the $r$-disk proximity neighbour set. This will be detailed in Section 4 .

Finally, in the problem, no specification is given for the trajectory $x(t)(t \in\{0,1, \ldots\})$ except for the convergence (7). This implies that, for instance, collisions among agents and roundabout trajectories are allowed in the solution. Such phenomena are not addressed in this paper in order to focus on the fundamental solution as the first step, while they have to be taken into account in practice and should be considered as the next step of this study.

\section{Consensus controllers}

In this section, we give a solution to Problem 1 .

The idea of the solution is outlined as follows. We assume that the local controllers $L_{i}(i=1,2, \ldots, N)$ are composed of two subcontrollers, as shown in Figure 5. The subcontrollers are given by

$$
\begin{aligned}
& L_{i 1}:\left\{\begin{aligned}
\xi_{i}(t+1) & =\alpha_{1}\left(\xi_{i}(t),\left\{v_{j}(t)\right\}_{j \in \mathbf{N}_{i}(t)}, w(t)\right) \\
u_{i 1}(t) & =\beta_{11}\left(\xi_{i}(t), w(t)\right) \\
v_{i}(t) & =\beta_{21}\left(\xi_{i}(t)\right)
\end{aligned}\right. \\
& L_{i 2}: u_{i 2}(t)=\beta_{12}\left(\left\{x_{j}(t)-x_{i}(t)\right\}_{j \in \mathbf{N}_{i}(t)}\right),
\end{aligned}
$$

where $u_{i 1}(t) \in \mathbf{R}^{n}$ and $u_{i 2}(t) \in \mathbf{R}^{n}$ are the outputs, and $\alpha_{1}$ : $\mathbf{R}^{v} \times \mathbf{P}\left(\mathbf{R}^{m}\right) \times \mathbf{R} \rightarrow \mathbf{R}^{v}, \beta_{11}: \mathbf{R}^{v} \times \mathbf{R} \rightarrow \mathbf{R}^{n}, \beta_{21}: \mathbf{R}^{v}$ $\rightarrow \mathbf{R}^{m}$, and $\beta_{12}: \mathbf{P}\left(\mathbf{R}^{n}\right) \rightarrow \mathbf{R}^{n}$ are functions. In addition, it is assumed that the agents are grouped according to a connection-based rule. Then, the controllers $L_{i 1}(i=1$, $2, \ldots, N)$ and $B$ are used for governing the group-to-group relation, while $L_{i 2}(i=1,2, \ldots, N)$ are for the agent-toagent relation in the groups. That is, we propose a controller set governing the global behaviour and the local behaviour separately.

\subsection{Group-to-group controllers}

For deriving controllers $L_{i 1}(i=1,2, \ldots, N)$ and $B$, we first introduce the notion of the group.

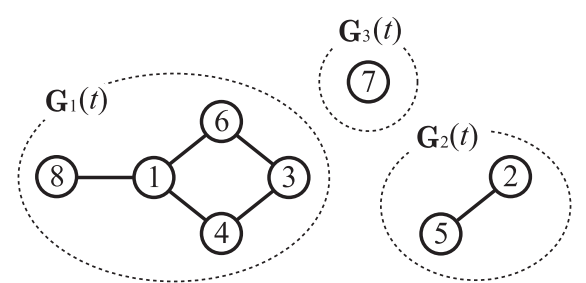

Figure 6. Example of groups $\mathbf{G}_{k}(t)(k=1,2, \ldots, M(t))$.

For $t \in\{0,1, \ldots\}$, let $M(t)$ and $\mathbf{G}_{1}(t), \mathbf{G}_{2}(t), \ldots, \mathbf{G}_{M(t)}(t)$ $\subseteq\{1,2, \ldots, N\}$ be the number and the index sets of agents such that

(i) $\bigcup_{k \in\{1,2, \ldots, M(t)\}} \mathbf{G}_{k}(t)=\{1,2, \ldots, N\}$, and $\mathbf{G}_{k}(t) \cap$ $\mathbf{G}_{l}(t)=\emptyset$ for every $(k, l) \in\{1,2, \ldots, M(t)\}^{2}$ satisfying $k \neq l$;

(ii) for each $k \in\{1,2, \ldots, M(t)\}$, the undirected graph with the node set $\mathbf{G}_{k}(t)$ and the edge set $\{(i, j) \in$ $\left.\mathbf{G}_{k}(t) \times \mathbf{G}_{k}(t) \mid i \in \mathbf{N}_{j}(t), i<j\right\}$ is connected;

(iii) $\left\{(i, j) \in \mathbf{G}_{k}(t) \times \mathbf{G}_{l}(t) \mid i \in \mathbf{N}_{j}(t)\right\}=\emptyset$ for every $(k, l) \in\{1,2, \ldots, M(t)\}^{2}$ satisfying $k \neq l$;

(iv) $\min \left(\mathbf{G}_{1}(t)\right)<\min \left(\mathbf{G}_{2}(t)\right)<\cdots<\min \left(\mathbf{G}_{M(t)}(t)\right)$.

The set $\mathbf{G}_{k}(t)$ is called the group (at time $t$ ), and $M(t)$ and $\mathbf{G}_{k}(t)(k=1,2, \ldots, M(t))$ are always uniquely determined. Roughly speaking, the group $\mathbf{G}_{k}(t)$ corresponds to an agent set in which each agent has a connection to the others. For example, in Figure 6, we have $M(t)=3, \mathbf{G}_{1}(t)=\{1,3$, $4,6,8\}, \mathbf{G}_{2}(t)=\{2,5\}$, and $\mathbf{G}_{3}(t)=\{7\}$. The conditions (i)-(iv), respectively, imply that the index set $\{1,2, \ldots, N\}$ of agents is divided into disjoint sets, the agents in each group are connected, two connected agents do not belong to different groups, and the groups $\mathbf{G}_{k}(t)(k=1,2, \ldots$, $M(t))$ are unique. that

Note in the system $\Sigma$ (with any controllers $L_{i}$ and $B$ )

$$
\begin{aligned}
M(t) & \geq M(t+1), \\
M\left(t_{1}\right)=M\left(t_{2}\right) \rightarrow \mathbf{G}_{k}\left(t_{1}\right) & =\mathbf{G}_{k}\left(t_{2}\right) \quad\left(k=1,2, \ldots, M\left(t_{1}\right)\right)
\end{aligned}
$$

for every $t \in\{0,1, \ldots\}$ and $\left(t_{1}, t_{2}\right) \in\{0,1, \ldots\}^{2}$. These are straightforward consequences of (5) and the definition of the groups. Also, note from (10) and the fact $M(t) \in\{1$, $2, \ldots, N\}$ that there exists a finite time $t \in\{0,1, \ldots\}$ such that

$$
M(t)=M(t+1)=\cdots=M(\infty) .
$$

The time $t$ depends on $r, L_{i}(i=1,2, \ldots, N), B$, and $x(0)$. 
Next, the time set $\{0,1, \ldots\}$ is divided into the disjoint sets

$$
\begin{aligned}
& \mathbf{T}_{1}:=\{0, N+3,(N+3) 2, \ldots\}, \\
& \mathbf{T}_{2}:=\mathbf{T}_{1} \oplus\{1,2, \ldots, N-1\}, \\
& \mathbf{T}_{3}:=\mathbf{T}_{1} \oplus\{N\}, \\
& \mathbf{T}_{4}:=\mathbf{T}_{1} \oplus\{N+1\}, \\
& \mathbf{T}_{5}:=\mathbf{T}_{1} \oplus\{N+2\} .
\end{aligned}
$$

These are convenient to express periodic switching controllers of period $N+3$.

Now, our controllers $L_{i 1}(i=1,2, \ldots, N)$ and $B$ are given by

$$
\begin{aligned}
& \alpha_{1}\left(\xi_{i}(t), \begin{cases}\left.\left\{v_{j}(t)\right\}_{j \in \mathbf{N}_{i}(t)}, w(t)\right) \\
{\left[\begin{array}{l}
\Delta_{i}(t) \\
w(t)
\end{array}\right]} & \text { if } t \in \mathbf{T}_{1} \\
{\left[\begin{array}{cc}
\operatorname{abmax}\left(\left\{\xi_{i 1}(t)\right\} \cup\left\{v_{j}(t)\right\}_{j \in \mathbf{N}_{i}(t)}\right) \\
\xi_{i 2}(t)
\end{array}\right]} & \text { if } t \in \mathbf{T}_{2} \\
{\left[\begin{array}{c}
\operatorname{sign}\left(\xi_{i 1}(t)\right) \\
\xi_{i 2}(t)
\end{array}\right]} & \text { if } t \in \mathbf{T}_{3} \\
{\left[\begin{array}{c}
\xi_{i 1}(t) \\
\xi_{i 2}(t)
\end{array}\right]} & \text { otherwise }\end{cases} \right.
\end{aligned}
$$

$$
\begin{aligned}
& \beta_{11}\left(\xi_{i}(t), w(t)\right) \\
& := \begin{cases}c(t) \xi_{i 1}(t) & \text { if } t \in \mathbf{T}_{4} \\
-c(t) \xi_{i 1}(t)-a(t) \frac{w(t)-\xi_{i 2}(t)}{c(t)} \xi_{i 1}^{(-1)}(t) & \text { if } t \in \mathbf{T}_{5}, \\
0 & \text { otherwise }\end{cases}
\end{aligned}
$$

$$
\beta_{21}\left(\xi_{i}(t)\right):=\xi_{i 1}(t)
$$

$$
\gamma(J(x(t))):=J(x(t))
$$

where $\xi_{i 1}(t) \in \mathbf{R}^{n}$ and $\xi_{i 2}(t) \in \mathbf{R}$ are the components of the state $\xi_{i}(t)$ such that

$$
\xi_{i}(t)=\left[\begin{array}{l}
\xi_{i 1}(t) \\
\xi_{i 2}(t)
\end{array}\right]
$$

$\xi_{i 1}^{(-1)}(t) \in \mathbf{R}^{n}$ is the elementwise inverse of $\xi_{i 1}(t)$ as defined in Section $1, \Delta_{i}(t) \in \mathbf{R}^{n}$ is a random vector whose elements are not zero w.p.1, and $a(t) \in \mathbf{R}_{+}$and $c(t) \in \mathbf{R}_{+}$are the time-varying gains of the controllers $L_{i 1}(i=1,2, \ldots, N)$.

The controllers $L_{i 1}(i=1,2, \ldots, N)$ play a role to steer the groups according to the broadcast signals. This is performed in five steps. Assume $t \in \mathbf{T}_{1}$. In Step 1 ( $t \in$ $\left.\mathbf{T}_{1}\right), L_{i 1}$ generates a random vector $\Delta_{i}(t)$ and receives the broadcast signal $w(t)$, which are, respectively, saved to $\xi_{i 1}(t$ $+1)$ and $\xi_{i 2}(t+1)$. Next, in Step $2(t+1, t+2, \ldots$, $\left.t+N-1 \in \mathbf{T}_{2}\right)$, it synchronises the random vectors in the same group by exchanging the information on $\Delta_{i}(t)$. Since $N$ agents are contained in the system $\Sigma$, this step is done in (at most) $N-1$ time steps. The synchronised random vector is quantised by the signum function in Step $3(t+$ $\left.N \in \mathbf{T}_{3}\right)$ and saved to $\xi_{i 1}(t+N+1)$. In Step $4(t+N$ $+1 \in \mathbf{T}_{4}$ ), it moves agent $i$ to a point determined by the synchronised vector. Finally, in Step $5\left(t+N+2 \in \mathbf{T}_{5}\right)$, it receives the broadcast signal $w(t+N+2)$ and generates an input to move agent $i$ to a point determined by the state $\xi_{i}(t+N+2)$ and the broadcast signal $w(t+N+2)$. In Steps 4 and 5, note that the agents in the same group move in the same direction and to the same distance. The above five steps are repeated for each $t \in \mathbf{T}_{1}$.

The global controller $B$, on the other hand, broadcasts the value of $J(x(t))$.

The following result is obtained for the controllers.

Lemma 1: For the system $\Sigma$, suppose that $r \in \boldsymbol{R}_{+}$and $x_{d}$ $\in \boldsymbol{R}^{n}$ are given. Suppose also that $L_{i l}(i=1,2, \ldots, N)$ and $B$ are given by (3), (8), (13), (14), (15), and (16), and $L_{i 2}$ $(i=1,2, \ldots, N)$ are arbitrarily given so as to satisfy

(A0) $\sum_{i \in \mathbf{G}_{k}(t)} u_{i 2}(t)=0(k=1,2, \ldots, M(t))$ for $t \in \boldsymbol{T}_{5}$ and $u_{i 2}(t)=0(i=1,2, \ldots, N)$ for $t \notin \boldsymbol{T}_{5}$.

Let $\tau \in\{0,1, \ldots\}$ be a time $t \in \boldsymbol{T}_{1}$ satisfying (12), and let $z_{k}(t) \in \boldsymbol{R}^{n}$ be the centroid of the positions $x_{i}(t)$ for $i \in \boldsymbol{G}_{k}(\tau)$, i.e.

$$
z_{k}(t):=\frac{1}{\left|\mathbf{G}_{k}(\tau)\right|} \sum_{i \in \mathbf{G}_{k}(\tau)} x_{i}(t)
$$

Let also $\Delta_{i j}(t)$ denote the jth element of $\Delta_{i}(t)$. If
(A1) $a(t)=a(t+1)=\cdots=a(t+N+2)$ and $c(t)$ $=c(t+1)=\cdots=c(t+N+2)$ for every $t \in \boldsymbol{T}_{1}$, $\lim _{t \rightarrow \infty} a(t)=0, \sum_{t=0}^{\infty} a(t)=\infty, \lim _{t \rightarrow \infty} c(t)=$ 0 , and $\sum_{t=0}^{\infty}(a(t) / c(t))^{2}<\infty$,

(A2) $\Delta_{i l}(t), \Delta_{i 2}(t), \ldots, \Delta_{i n}(t)(i=1,2, \ldots, N, t=0$, $1, \ldots)$ are i.i.d. random variables from a probability distribution which is continuous and symmetric about zero (and $\Delta_{i j}(t) \neq 0$ w.p. 1 as stated above),

then

$$
\lim _{t \rightarrow \infty} z_{k}(t)=x_{d} \text { w.p. } 1
$$

for every $k \in\{1,2, \ldots, M(\tau)\}$.

Proof: Let $\mathbf{T}_{1}(\tau):=\mathbf{T}_{1} \cap\{\tau, \tau+1, \ldots\}$ and $z(t):=\left[z_{1}^{\top}(t) z_{2}^{\top}(t) \cdots z_{M(\tau)}^{\top}(t)\right]^{\top} \in \mathbf{R}^{n M(\tau)}$. Then, (19) is a straightforward consequence of the following three facts (a), (b), and (c) which are proved in Appendix B. 
(a) If (A0) and (A1) hold,

$$
\begin{aligned}
& z(t+N+3) \\
& =z(t)-a(t) \frac{J_{c}(z(t)+c(t) \Delta(t))-J_{c}(z(t))}{c(t)} \Delta^{(-1)}(t)
\end{aligned}
$$

for every $t \in \mathbf{T}_{1}(\tau)$, where

$$
\begin{gathered}
\Delta(t):=\left[\begin{array}{c}
\operatorname{sign}\left(\operatorname{abmax}\left(\left\{\Delta_{j}(t)\right\}_{\left.j \in \mathbf{G}_{1}(\tau)\right)}\right)\right. \\
\operatorname{sign}\left(\operatorname{abmax}\left(\left\{\Delta_{j}(t)\right\}_{j \in \mathbf{G}_{2}(\tau)}\right)\right) \\
\vdots \\
\operatorname{sign}\left(\operatorname { a b m a x } \left(\left\{\Delta_{j}(t)\right\}_{\left.\left.j \in \mathbf{G}_{M(\tau)}(\tau)\right)\right)}\right.\right.
\end{array}\right], \\
J_{c}(z(t)):=\sum_{k=1}^{M(\tau)}\left|\mathbf{G}_{k}(\tau)\right|\left\|z_{k}(t)-x_{d}\right\|^{2} .
\end{gathered}
$$

Note in (20) that $t+N+3 \in \mathbf{T}_{1}(\tau)$ for $t \in \mathbf{T}_{1}(\tau)$.

(b) If (A1) and (A2) hold, the dynamics in (20) corresponds to the stochastic approximation algorithm in (A.1) (in Appendix A), which converges to a local minimum point of the function $F$ w.p.1, by regarding $z, \Delta, J_{c}$, $t$, and $t+N+3$ as $\zeta, \theta, F, k$, and $k+1$, respectively.

(c) The function $J_{c}$ has the unique local minimum point at $\left[\begin{array}{lll}x_{d}^{\top} & x_{d}^{\top} \cdots x_{d}^{\top}\end{array}\right]^{\top}$, and $\lim _{t \rightarrow \infty} J_{c}(z(t))=0$ w.p.1 implies that (19) holds for every $k \in\{1,2, \ldots$, $M(\tau)\}$.

From Lemma 1, it turns out that the proposed controllers $L_{i 1}(i=1,2, \ldots, N)$ and $B$ attain the consensus for the groups formed at time $\tau$. The idea behind this result is as follows. As shown in the proof, the groups (the centroids $\left.z_{k}(k=1,2, \ldots, M(\tau))\right)$ evolve according to (20) for $t \in$ $\mathbf{T}_{1}(\tau)$. Then, for the second term on the right-hand side of (20), it can be shown by a simple calculation that

$$
\begin{gathered}
\mathbb{E}\left(a(t) \frac{J_{c}(z(t)+c(t) \Delta(t))-J_{c}(z(t))}{c(t)} \Delta^{(-1)}(t) \mid z(t)\right) \\
\quad=a(t)\left(\nabla J_{c}(z(t))+O(c(t))\right) \quad(c(t) \rightarrow 0)
\end{gathered}
$$

where $O(c(t))$ is a function of $c(t)$ satisfying $\lim _{c(t) \rightarrow 0} O(c(t)) / c(t)<\infty$. So, it follows from (A1) $(c(t) \rightarrow$ $0)$ that (20) corresponds to $\mathbb{E}(z(t+N+3) \mid z(t)) \simeq z(t)-$ $a(t) \nabla J_{c}(z(t))$, i.e. a stochastic version of the gradientdescent for $J_{c}$. This fact implies that $z(t)$ converges to a local minimum point of $J_{c}$. Meanwhile, as seen in Fact (c) in the proof, $J_{c}$ has the unique local minimum point at $\left[x_{d}^{\top} x_{d}^{\top} \cdots x_{d}^{\top}\right]^{\top}$. These provide (19).

Next, let us comment on the conditions (A1) and (A2). The conditions are given for the parameters designed by users, i.e. the gains $a(t)$ and $c(t)$ and the probability distribution of $\Delta_{i}(t)$. The conditions for $a(t)$ in (A1) mean that $a(t)$ changes every $N+3$ steps and $a(t)$ decreases neither too fast nor too slow in order to avoid precocious convergence in the early stage of the control and to get fast convergence in the final stage. The conditions for $c(t)$ and for both $a(t)$ and $c(t)$ are technical ones to guarantee the almost-sure convergence in (19), which imply that $c(t)$ also changes every $N+3$ steps and $c(t)$ converges to zero but more slowly than $a(t)$. Examples of $a(t)$ and $c(t)$, which satisfy (A1), are shown in Section 3.4. On the other hand, (A2) specifies the probability distribution for $\Delta(t)$. For example, it is satisfied if each element of $\Delta(t)$ is drawn from the uniform distribution on $[-1,1]$. It should be noticed that the choice of the probability distribution does not affect the controller output $u_{i 1}$, because, for any probability distribution satisfying (A2), each element of $\xi_{i 1}(t+1)$ for $t \in \mathbf{T}_{3}$ is equivalent to the random variables drawn from the Bernoulli distribution with outcome \pm 1 and equal probabilities (see (8) and (13)).

\subsection{Agent-to-agent controllers}

Next, the controllers $L_{i 2}(i=1,2, \ldots, N)$ are given by

$$
\begin{aligned}
\beta_{12}\left(\left\{x_{j}(t)-x_{i}(t)\right\}_{j \in \mathbf{N}_{i}(t)}\right) \\
:= \begin{cases}\varepsilon \sum_{j \in \mathbf{N}_{i}(t)}\left(x_{j}(t)-x_{i}(t)\right) & \text { if } t \in \mathbf{T}_{5} \\
0 & \text { otherwise }\end{cases}
\end{aligned}
$$

where $\varepsilon \in \mathbf{R}_{+}$is the gain of the controllers. The above controllers work as a standard consensus controller based on the relative positions to the neighbours (see, e.g. OlfatiSaber et al., 2007) at time $t \in \mathbf{T}_{5}$ and do not work at time $t \notin \mathbf{T}_{5}$. So, the agents in each group are expected to approach each other at $t \in \mathbf{T}_{5}$. This is formalised as follows.

Lemma 2: For the system $\Sigma$, suppose that $r \in \boldsymbol{R}_{+}$is given. Suppose also that $L_{i 2}(i=1,2, \ldots, N)$ are given by (9) and (24), $L_{i 1}(i=1,2, \ldots, N)$ are arbitrarily given so as to satisfy

(B0) for every $t \in\{0,1, \ldots\}$ and $k \in\left\{1,2, \ldots, M\left(t_{1}\right)\right\}$,

$$
u_{i 1}(t)=u_{j 1}(t) \quad\left(\forall(i, j) \in \mathbf{G}_{k}\left(t_{1}\right) \times \mathbf{G}_{k}\left(t_{1}\right)\right),
$$

where $t_{1}:=\lfloor t\rfloor_{\mathbf{T}_{1}}$

and $B$ is arbitrarily given. If

(B1) $0<\varepsilon<1 / N$,

then

$$
\lim _{t \rightarrow \infty} x_{i}(t)-x_{j}(t)=0 \quad\left(\forall(i, j) \in \mathbf{G}_{k}(s) \times \mathbf{G}_{k}(s)\right)
$$

for every $s \in\{0,1, \ldots\}$ and $k \in\{1,2, \ldots, M(s)\}$. 
Proof: Let $\tau \in\{0,1, \ldots\}$ be time $t \in \mathbf{T}_{1}$ satisfying $s \leq t$ and (12).

First, consider the dynamics of the agents in $\mathbf{G}_{l}(\tau)$ for $l$ $\in\{1,2, \ldots, M(\tau)\}$. By the definition of $\tau$ and (11), we have $\mathbf{G}_{l}(\tau)=\mathbf{G}_{l}(\tau+1)=\cdots=\mathbf{G}_{l}(\infty)$. Using this property with (1), (9), (24), (B0), and Figure 5, we obtain

$$
\begin{aligned}
x_{(l)}( & +N+3) \\
= & x_{(l)}(t)-\varepsilon\left(L_{(l)} \otimes I_{n}\right) x_{(l)}(t+N+2) \\
& +\mathbf{1}_{\left|\mathbf{G}_{l}(\tau)\right|} \otimes\left(u_{i 1}(t)+u_{i 1}(t+1)\right. \\
& \left.+\cdots+u_{i 1}(t+N+2)\right) \\
= & x_{(l)}(t)-\varepsilon\left(L_{(l)} \otimes I_{n}\right) x_{(l)}(t)+\mathbf{1}_{\left|\mathbf{G}_{l}(\tau)\right|} \otimes\left(u_{i 1}(t)\right. \\
& \left.+u_{i 1}(t+1)+\cdots+u_{i 1}(t+N+2)\right)
\end{aligned}
$$

for $t \in \mathbf{T}_{1}(\tau)$ and $i \in \mathbf{G}_{l}(\tau)$, where $x_{(l)}(t) \in \mathbf{R}^{n\left|\mathbf{G}_{l}(\tau)\right|}$ is the vector composed of $x_{i}(t)$ for $i \in \mathbf{G}_{l}(\tau)$ and $L_{(l)} \in$ $\mathbf{R}^{\left|\mathbf{G}_{l}(\tau)\right| \times\left|\mathbf{G}_{l}(\tau)\right|}$ is the graph Laplacian for the agents in $\mathbf{G}_{l}(\tau)$. Note here that $t+N+3 \in \mathbf{T}_{1}(\tau)$ for $t \in \mathbf{T}_{1}(\tau)$. If (B1) holds (under which the corresponding Perron matrix is primitive), (26) is a discrete-time consensus algorithm (see, e.g. Olfati-Saber et al., 2007), i.e. $\lim _{\mu \rightarrow \infty} x_{i}((N+$ 3) $\mu)-x_{j}((N+3) \mu)=0$ for every $(i, j) \in \mathbf{G}_{l}(\tau) \times \mathbf{G}_{l}(\tau)$, where $\mu \in\{0,1, \ldots\}$. In addition, (1), (9), (24), (B0), and Figure 5 provide $x_{i}(t)-x_{j}(t)=x_{i}(t+1)-x_{j}(t+1)=$ $\cdots=x_{i}(t+N+2)-x_{j}(t+N+2)$ for every $t \in \mathbf{T}_{1}(\tau)$ and $(i, j) \in \mathbf{G}_{l}(\tau) \times \mathbf{G}_{l}(\tau)$. So, $\lim _{t \rightarrow \infty} x_{i}(t)-x_{j}(t)=0$ for every $(i, j) \in \mathbf{G}_{l}(\tau) \times \mathbf{G}_{l}(\tau)$.

Meanwhile, it is straightforward by $s \leq \tau$ and the definition of the groups that, for each $k \in\{1,2, \ldots, M(s)\}$, there exists an $l \in\{1,2, \ldots, M(\tau)\}$ such that $\mathbf{G}_{k}(s) \subseteq \mathbf{G}_{l}(\tau)$.

The above two facts prove (25).

This lemma shows that the controllers $L_{i 2}(i=1,2, \ldots$, $N)$ achieve the local consensus in the groups for any time.

\subsection{Convergence result}

From (8), (9), (13), (14), (15), (24), and the fact that

$$
\xi_{i 1}(t)=\xi_{j 1}(t)\left(\forall(i, j) \in \mathbf{G}_{k}(t) \times \mathbf{G}_{k}(t)\right)
$$

for $t \in \mathbf{T}_{4} \cup \mathbf{T}_{5}$ (which is given by Lemma 4 in Appendix $\mathrm{B})$, it is clear that (A0) and (B0) hold for the proposed controllers $L_{i 1}$ and $L_{i 2}(i=1,2, \ldots, N)$. So, combining Lemmas 1 and 2, we obtain a solution to Problem 1 .

Theorem 1: For the system $\Sigma$, suppose that $r \in \boldsymbol{R}_{+}$and $x_{d} \in \boldsymbol{R}^{n}$ are given. Let $L_{i 1}, L_{i 2}(i=1,2, \ldots, N)$, and $B$ be given by (3), (8), (9), (13), (14), (15), (16), and (24). If (A1), (A2), and (B1) hold, (7) holds w.p.1.

Two remarks are given.

First, this theorem shows that the proposed controller is an almost-sure solution due to the stochastic controllers $L_{i 1}(i=1,2, \ldots, N)$ (which include the random variables
$\left.\Delta_{i}(t)\right)$. Such a solution is quite reasonable in our problem setting because, as shown in Azuma et al. (2013), the use of stochastic controllers is essential in the broadcast-based multi-agent control.

Second, conditions (A1), (A2), and (B1) are imposed for the tuning parameters of $L_{i 1}$ and $L_{i 2}$. Thus, they are not restrictive in practice.

\subsection{Example}

Consider the system $\Sigma$ with $N:=20$ and $n:=2$. The neighbour sets $\mathbf{N}_{i}(t)(i=1,2, \ldots, 20)$ are defined for $r:=$ 0.25 and the desired location $x_{d}$ is assumed to be the origin, i.e. $x_{d}:=0$.

We use the controllers $L_{i}(i=1,2, \ldots, 20)$ and $B$ given in Section 3. The gains of $L_{i 1}$ are given by

$$
a(t):=\frac{0.07}{\left(\frac{t}{N+3}+22\right)^{0.7}}, \quad c(t):=\frac{0.01}{\left(\frac{t}{N+3}+1\right)^{0.16}}
$$

for $t \in \mathbf{T}_{1}$ and $a(t):=a(t-1)$ and $c(t):=c(t-1)$ for $t \notin \mathbf{T}_{1}$. They are selected according to the guideline (Spall, 2003) for the simultaneous perturbation stochastic approximation, by considering Fact (b) in the proof of Lemma 1. Each element of $\Delta_{i}(t)(t=0,1, \ldots)$ is drawn from the uniform distribution on $[-1,1]$, which is a standard distribution as stated in Section 3.1. The gain $\varepsilon$ of $L_{i 2}$ is set as $\varepsilon:=0.03$. Then, (A1), (A2), and (B1) in Theorem 1 hold.

Figure 7 illustrates the snapshots of the agent positions $x_{i}(t)$ at $t=0,500, \ldots, 2500$, where the circles express the agents and the solid lines, connecting circles, represent the relation $j \in \mathbf{N}_{i}(t)$. Figure 8, on the other hand, exhibits the time evolution of the function $J(x(t))$ (by the thick line). These show that the proposed controllers achieve the consensus at the origin via the consensus among the groups and that in the groups.

For comparison, the existing broadcast control technique (Azuma et al., 2013) is applied to the same system assuming that only the broadcast can be used (i.e. without communication). The thin line in Figure 8 depicts the time evolution of $J(x(t))$. We see that the convergence is much slower than that by the proposed controllers, that is, higher performance is realised under the mixed environment of communication and broadcast.

Finally, we discuss the performance of the proposed controller under several conditions. Table 1 shows the relation between the number $N$ of agents and the convergence time, where $\varepsilon:=1 /(N+1)$ (which satisfies (B1)) and the other conditions are the same as above. Here, the convergence time means the time $t$ required for the value of $J(x(t))$ to fall to the $10 \%$ value of $J(x(0))$ (more precisely, the minimum $t \in\{0,1, \ldots\}$ such that $J(x(t)) \leq 0.1 J(x(0)))$. In the table, it is seen that the convergence time grows with the number $N$, which is the same property as observed for 


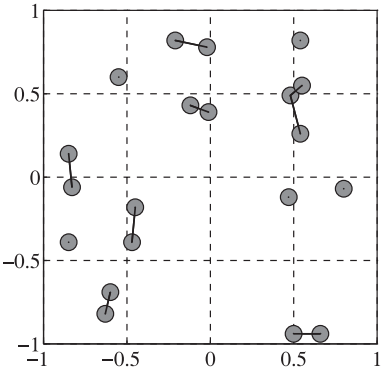

(a) $t=0$.

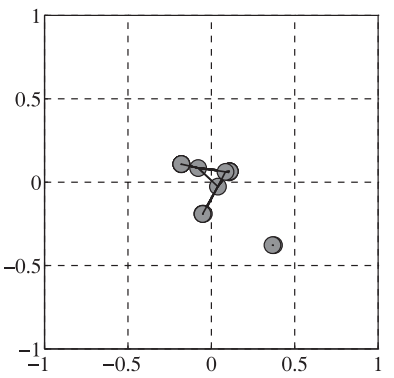

(c) $t=1000$

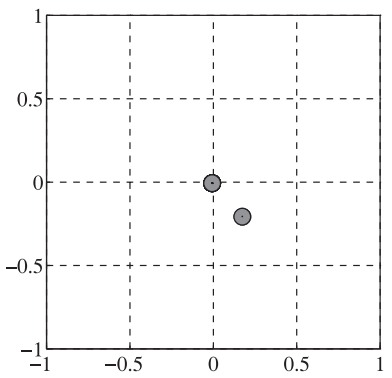

(e) $t=2000$

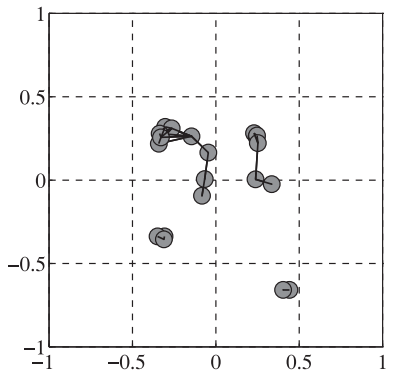

(b) $t=500$

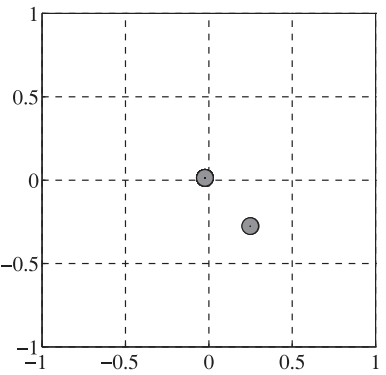

(d) $t=1500$

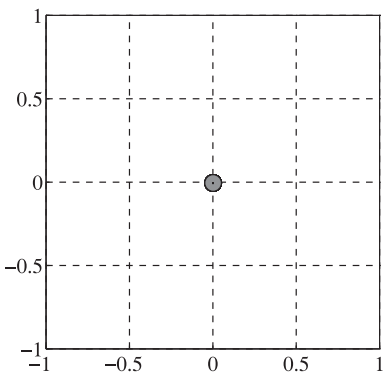

(f) $t=2500$
Figure 7. Time evolution of agent positions by the proposed consensus controller $\left(N=20, n=2, r=0.25\right.$, and $\left.x_{d}=0\right)$. (a) $t=0$. (b) $t=500$. (c) $t=1000$. (d) $t=1500$. (e) $t=2000$. (f) $t=2500$.

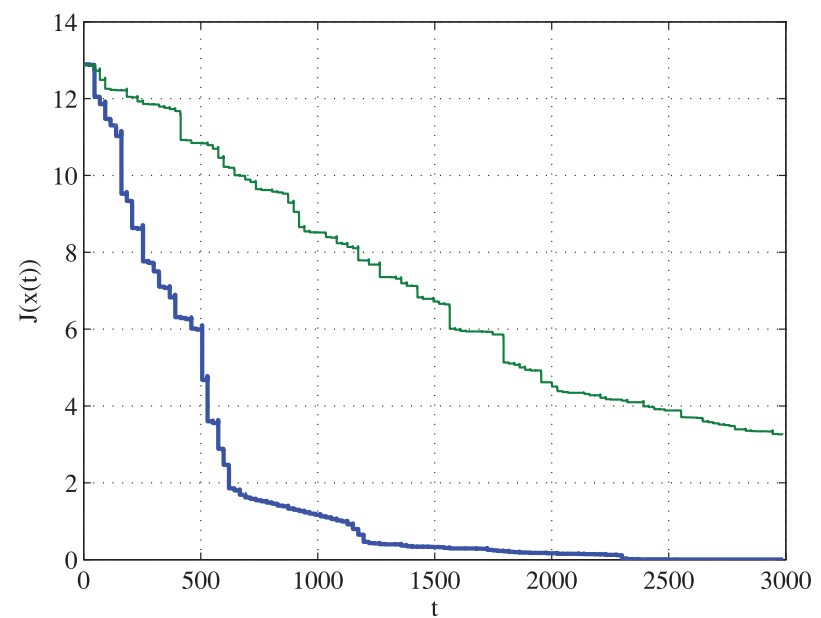

Figure 8. Time evolution of achievement degree $J(x(t))$ (thick line: proposed consensus controller; thin line: simple broadcastbased consensus controller in Azuma et al., 2013).
Table 1. Relation between the number $N$ of agents and convergence time.

\begin{tabular}{lcrrr}
\hline Number $N$ of agents & & 10 & 20 & 40 \\
\hline Convergence time (s) & Maximum & 1469 & 1978 & 12599 \\
(based on 10 trials) & Mean & 950 & 1576 & 9138 \\
& Minimum & 364 & 782 & 2924 \\
\hline
\end{tabular}

many communication-based consensus controllers and the broadcast control in Azuma et al. (2013). Next, Table 2 shows the convergence times for several initial configurations $x(0)$, where the initial positions $x_{i}(0)(i=1,2, \ldots$, $N)$ are randomly generated by the uniform distribution on the spaces $[-0.5,0.5]^{2},[-1,1]^{2}$, and $[-2,2]^{2}$. The other conditions are the same as above. In the table, it turns out that the convergence time increases as the dispersion of the initial positions increases. This is a reasonable result for the consensus problem because the consensus is regarded as minimising the spatial dispersion.

\section{Extension to the case with an $r$-disk proximity neighbour set}

The proposed framework can be extended to the case where the neighbours are defined by the spatial proximity property.

Consider the feedback system $\Sigma$ in Figure 3 in the continuous-time domain. Let $t_{c} \in \mathbf{R}_{0}+$ be the continuoustime variable. We use here the same symbols as introduced in Sections 2 and 3, but the signals, the time-varying sets, and the time-varying gains are denoted with the brackets \langle\rangle , in order to distinguish from those in the discrete-time domain. For example, $x_{i}\left\langle t_{c}\right\rangle$ represents the position of agent $i$ in the continuous-time domain. The neighbour set considered here is given by

$\mathbf{N}_{i}\left\langle t_{c}\right\rangle:=\left\{j \in\{1,2, \ldots, N\} \backslash\{i\} \mid\left\|x_{j}\left\langle t_{c}\right\rangle-x_{i}\left\langle t_{c}\right\rangle\right\|<r\right\}$,

for which the groups $\mathbf{G}_{k}\left\langle t_{c}\right\rangle\left(k=1,2, \ldots, M\left\langle t_{c}\right\rangle\right)$ and the number $M\left\langle t_{c}\right\rangle$ are defined in the same way.

A solution to the continuous-time version of Problem 1 is given as follows. Assume that the physical dynamics of agent $i$ is given by

$$
P_{i}: \dot{x}_{i}\left\langle t_{c}\right\rangle=u_{i}\left\langle t_{c}\right\rangle
$$

and the local controllers $L_{i}(i=1,2, \ldots, N)$ are in the form of Figure 5 . The subcontrollers $L_{i 1}(i=1,2, \ldots, N)$ and $B$ are given by 
Table 2. Relation between the initial configuration and convergence time.

\begin{tabular}{lcccc}
\hline Space for initial configuration & {$[-0.5,0.5]^{2}$} & {$[-1,1]^{2}$} & {$[-2,2]^{2}$} \\
\hline Convergence time (s) & Maximum & 1863 & 2703 & 3611 \\
(based on 10 trials) & Mean & 1145 & 1519 & 2242 \\
& Minimum & 575 & 552 & 1380 \\
\hline
\end{tabular}

$$
L_{i 1}:\left\{\begin{array}{c}
\xi_{i}\langle h(t+1)\rangle=\alpha_{1}\left(\xi_{i}\langle h t\rangle,\left\{v_{j}\langle h t\rangle\right\}_{j \in \mathbf{N}_{i}\langle h t\rangle}, w\langle h t\rangle\right) \\
u_{i 1}\left\langle t_{c}\right\rangle=\frac{1}{h} \beta_{11}\left(\xi_{i}\langle h t\rangle, w\langle h t\rangle\right) \\
\left(\forall t_{c} \in[h t, h(t+1))\right) \\
v_{i}\left\langle t_{c}\right\rangle=\frac{1}{h} \beta_{21}\left(\xi_{i}\langle h t\rangle\right)\left(\forall t_{c} \in[h t, h(t+1))\right)
\end{array},\right.
$$

$B: w\left\langle t_{c}\right\rangle=\gamma(J(x\langle h t\rangle))\left(\forall t_{c} \in[h t, h(t+1))\right)$

where $h \in \mathbf{R}_{+}$is the sampling period, $t \in\{0,1, \ldots\}$ is the sampling number (i.e. $h t(t=0,1, \ldots)$ are the sampling times), and $\alpha_{1}, \beta_{11}, \beta_{21}$, and $\gamma$ are given by (13), (14), (15), and (16), respectively, but where ' $(t)$ ' is replaced with ' $\langle h t\rangle$ '. On the other hand, the subcontrollers $L_{i 2}(i=1,2, \ldots, N)$ are given by

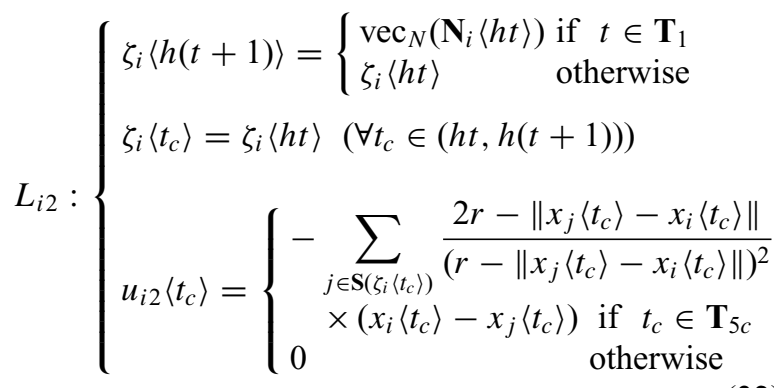

where $\zeta_{i}\left\langle t_{c}\right\rangle \in \mathbf{R}^{N}$ is the state (i.e. a memory) and $\mathbf{T}_{5 c}:=$ $\{h((N+3)-1), h((N+3) 2-1), h((N+3) 3-1), \ldots\}$ $\oplus[0, h)$. The subcontrollers $L_{i 2}$ are based on the consensus controller developed in Ji and Egerstedt (2007). Note in (32) that $\mathbf{S}\left(\zeta_{i}\left\langle t_{c}\right\rangle\right) \subseteq \mathbf{N}_{i}\left\langle t_{c}\right\rangle$ as shown later and so $u_{i 2}\left\langle t_{c}\right\rangle$ depends only on the information of the neighbours of agent $i$.

Then, the following result is obtained.

Theorem 2: For the feedback system $\Sigma$ in the continuoustime domain, suppose that $r \in \boldsymbol{R}_{+}$and $x_{d} \in \boldsymbol{R}^{n}$ are given. Assume that the neighbour set is given by (28) and let $L_{i 1}$, $L_{i 2}(i=1,2, \ldots, N)$, and $B$ be given in the above way. Then, the following statements hold.

(i) $\boldsymbol{S}\left(\zeta_{i}\left\langle t_{c}\right\rangle\right) \subseteq \boldsymbol{N}_{i}\left\langle t_{c}\right\rangle$ holds for every $t_{c} \in \boldsymbol{R}_{0+}$ (i.e. $L_{i 2}$ are distributed controllers with the information of their neighbours).

(ii) If
$\left(\mathrm{Al}^{\prime}\right)$ the continuous-time version of (A1), which is given by replacing ' $(t)$ ', ' $(t+1)$ ', ..., ' $(t+N+2)$ 'with ' $\langle h t\rangle$ ', ' $\langle h(t+1)\rangle$ ', ... ' $\langle h(t+N+2)\rangle$ ' in (A1),

$\left(\mathrm{A} 2^{\prime}\right)$ the continuous-time version of (A2), which is given by replacing ' $(t)$ ' with ' $\langle h t\rangle$ ' in (A2)

hold, then

$$
\lim _{t \rightarrow \infty} J(x\langle h t\rangle)=0 \text { w.p. } 1 .
$$

Proof. Statement (i) is proved in Appendix C2.

On the other hand, (ii) can be proved in the same way as Theorem 1 but with the following facts (which are proved in Appendix C1).

(a) For every $i \in\{1,2, \ldots, N\}$ and $t \in \mathbf{T}_{1}$,

$$
\mathbf{N}_{i}\langle h t\rangle \subseteq \mathbf{N}_{i}\left\langle t_{c}\right\rangle \quad\left(\forall t_{c} \in[h t, h(t+N+3)]\right) .
$$

(b) For the subcontrollers $L_{i 2}(i=1,2, \ldots, N)$,

$$
\begin{aligned}
\left(\mathrm{A}^{\prime}\right) & \sum_{i \in \mathbf{G}_{k}\left\langle h t_{1}\right\rangle} u_{i 2}\left\langle t_{c}\right\rangle=0\left(k=1,2, \ldots, M\left\langle h t_{1}\right\rangle\right) \\
& \text { for } t_{c} \in \mathbf{T}_{5 c}, \text { and } u_{i 2}\left\langle t_{c}\right\rangle=0(i=1,2, \ldots, \\
& N) \text { for } t_{c} \notin \mathbf{T}_{5 c}, \text { where } t_{1}:=\left\lfloor t_{c} / h\right\rfloor \mathbf{T}_{1}
\end{aligned}
$$

holds.

(c) For every $s \in \mathbf{T}_{1}$ and $k \in\{1,2, \ldots, M\langle h s\rangle\}$,

$$
\lim _{t \rightarrow \infty} x_{i}\langle h t\rangle-x_{j}\langle h t\rangle=0\left(\forall(i, j) \in \mathbf{G}_{k}\langle h s\rangle \times \mathbf{G}_{k}\langle h s\rangle\right) .
$$

In fact, since $\mathbf{N}_{i}\left\langle t_{c}\right\rangle$ is a finite set and $t+N+3 \in \mathbf{T}_{1}$ for $t \in \mathbf{T}_{1}$, (a) implies that there exists a $t \in \mathbf{T}_{1}$ such that

$$
\begin{aligned}
& \mathbf{N}_{i}\langle h t\rangle=\mathbf{N}_{i}\langle h(t+N+3)\rangle=\mathbf{N}_{i}\langle h(t+(N+3) 2)\rangle \\
& =\cdots=\mathbf{N}_{i}\langle\infty\rangle .
\end{aligned}
$$

From this, (30), and (31), it turns out that $L_{i 1}$ and $B$ are equivalent to those in Section 3.1 under the correspondence between time $h t$ in the continuous-time domain and time $t$ in the discrete-time domain. Thus, it follows from Lemma 1 that, if (b), ${ }^{1}$ (A1'), and (A2') hold, $\lim _{t \rightarrow \infty} z_{k}\langle h t\rangle=x_{d}$ w.p. 1 for every $k \in\{1,2, \ldots, M\langle h \tau\rangle\}$, where $\tau \in\{0,1$, $\ldots\}$ is a $t \in \mathbf{T}_{1}$ satisfying (35). This convergence and (c) establish (33). 


\section{Conclusion}

This paper has presented a consensus control method under a mixed environment of communication and broadcast. By decomposing the consensus problem into a global problem and a local problem, we have derived a consensus controller utilising the broadcast and the communication. The almostsure convergence for the proposed controller has been also proved. Finally, it has been shown by numerical simulation that the consensus is satisfactorily achieved by the proposed controller.

As the first step to multi-agent control under the mixed environment, this paper has focused on a consensus problem. In the future, this framework should be extended to other multi-agent problems such as the coverage problem and the formation problem. Moreover, it is expected to extend the results to a more general class of agents, such as with non-holonomic dynamics.

\section{Acknowledgements}

This work was partly supported by the Aihara Project, the FIRST programme from JSPS, initiated by CSTP.

\section{Note}

1. One may consider that $\left(\mathrm{A}^{\prime}{ }^{\prime}\right)$ is inconsistent with (A0) because the summation parts (the sets to which $i$ belongs) are slightly different. However, it is clear in (A0) that $\mathbf{G}_{k}(t)=\mathbf{G}_{k}\left(\lfloor t\rfloor_{\mathbf{T}_{1}}\right)$ for $t \in\{\tau, \tau+1, \ldots\}$ because of (11), (12), and the definition of $\tau$. In this sense, $\left(\mathrm{A}^{\prime}\right)$ is consistent with (A0). Note here that (A0) is used only for $t \in\{\tau, \tau+1, \ldots\}$ in the proof of Lemma 1.

\section{References}

Abdallah, C.T., \& Tanner, H.G. (2007). Complex networked control systems: Introduction to the special section. Control Systems Magazine, 27(4), 30-32.

Azuma, S., Baba, I., \& Sugie, T. (2012). Broadcast control of group of Markov chains. In 51st IEEE Conference on Decision and Control (pp. 2059-2064). Maui, HI.

Azuma, S., Selman Sakar, M., \& Pappas, G.J. (2012). Stochastic source seeking by mobile robots. IEEE Transactions on Automatic Control, 57(9), 2308-2321.

Azuma, S., Tanaka, Y., \& Sugie, T. (2012). Multi-agent consensus under communication-broadcast mixed environment. In 51st IEEE Conference on Decision and Control (pp. 94-99). Maui, HI.

Azuma, S., Yoshimura, R., \& Sugie, T. (2013). Broadcast control of multi-agent systems. Automatica, 49(8), 2307-2316.

Borkar, V.S. (2008). Stochastic approximation: A dynamical systems viewpoint. Cambridge: Cambridge University Press.

Bretl, T. (2007). Control of many agents using few instructions. In 2007 Proceedings of Robotics: Science and Systems Conference. Atlanta, GA.

Das, K., \& Ghose, D. (2009). Positional consensus in multi-agent systems using a broadcast control mechanism. In 2009 American Control Conference (pp. 5731-5736). St. Louis, MO.

Ji, M., \& Egerstedt, M. (2007). Distributed coordination control of multiagent systems while preserving connectedness. IEEE Transactions on Robotics, 23(4), 693-703.
Julius, A.A., Halasz, A., Sakar, M.S., Rubin, H., Kumar, V., \& Pappas, G.J. (2008). Stochastic modeling and control of biological systems: The lactose regulation system of Escherichia coli. IEEE Transactions on Automatic Control, 53(1), 51-65.

Martinez, S., Cortés, J., \& Bullo, F. (2007). Motion coordination with distributed information. Control Systems Magazine, 27(4), 75-88

Mesbahi, M., \& Egerstedt, M. (2010). Graph theoretic methods for multiagent networks. Princeton, NJ: Princeton University Press.

Olfati-Saber, R., Fax, J.A., \& Murray, R.M. (2007). Consensus and cooperation in networked multi-agent systems. Proceedings of the IEEE, 95(1), 215-233.

Spall, J.C. (1992). Multivariate stochastic approximation using a simultaneous perturbation gradient approximation. IEEE Transactions on Automatic Control, 37(3), 332-341.

Spall, J.C. (2003). Introduction to stochastic search and optimization. Hoboken, NJ: Wiley-Interscience.

Sundaram, S., \& Hadjicostis, C.N. (2008). Distributed function calculation and consensus using linear iterative strategies IEEE Journal on Selected Areas in Communications: Issue on Control and Communications, 26(4), 650-660.

Ueda, J., Odhner, L., \& Asada, H.H. (2007). Broadcast feedback of stochastic cellular actuators inspired by biological muscle control. International Journal of Robotics Research, 26(1112), 1251-1265.

Wood, L.B., Das, A., \& Asada, H.H. (2008). Broadcast feedback control of cell populations using stochastic Lyapunov functions with application to angiogenesis regulation. In 2008 American Control Conference (pp. 2105-2111). Seattle, WA

\section{Appendix A}

\section{One-sided simultaneous perturbation stochastic approximation}

Consider the system

$$
\zeta(k+1)=\zeta(k)-a(k) \frac{F(\zeta(k)+c(k) \theta(k))-F(\zeta(k))}{c(k)} \theta^{(-1)}(k)
$$

where $\zeta(k) \in \mathbf{R}^{p}$ is the state, $\theta(k) \in \mathbf{R}^{p}$ is a random vector whose elements are not zero w.p. $1, a(k) \in \mathbf{R}_{+}$and $c(k) \in \mathbf{R}_{+}$are the (time-varying) gains, $k \in\{0,1, \ldots\}$ is the time, and $F: \mathbf{R}^{p} \rightarrow \mathbf{R}$ is a function. The system (A.1) is a variety (a one-sided version) of the simultaneous perturbation stochastic approximation originally proposed in Spall (1992).

The convergence result (Azuma, Selman Sakar, \& Pappas, 2012) is summarised as follows.

Lemma 3: For the system (A.1), assume that $F$ is differentiable and there exists a vector $\zeta^{*} \in \boldsymbol{R}^{p}$ satisfying $\nabla F\left(\zeta^{*}\right)=0$. Let $\theta_{i}(k)$ $\in \boldsymbol{R}$ be the ith element of $\theta(k)$. If

(C1) F is twice differentiable (in addition to the differentiability assumed above)

(C2) $\zeta^{*}$ is an asymptotically stable equilibrium of the gradient system $\dot{\eta}(s)=-\nabla F(\eta(s))$, where $s \in \boldsymbol{R}_{0+}, \eta(s) \in \boldsymbol{R}^{p}$, and the stability is in the Lyapunov sense,

(C3) $\lim _{k \rightarrow \infty} a(k)=0, \sum_{k=0}^{\infty} a(k)=\infty, \lim _{k \rightarrow \infty} c(k)=0$, and $\sum_{k=0}^{\infty}(a(k) / c(k))^{2}<\infty$,

(C4) $\theta_{i}(k)(i=1,2, \ldots, p, k=0,1, \ldots)$ are i.i.d. random variables (independent also of $\zeta(k)(k=0,1, \ldots)$ from a probability distribution which is symmetry about 
zero, and there exists a $\bar{\theta} \in \mathbf{R}_{0+}$ such that $\left|\theta_{i}(k)\right| \leq \bar{\theta}$, $\left|\theta_{i}^{-1}(k)\right| \leq \bar{\theta}$, and $\left|\theta_{i}^{-2}(k)\right| \leq \bar{\theta}$ w.p. 1 ,

(C5) $\sup _{k \in\{0,1, \ldots\}}\|\zeta(k)\|<\infty$ w.p. 1 ,

(C6) $E\left[F(\zeta(k)+c(k) \theta(k))^{2}\right]$ is bounded for every $k \in\{0,1$, $\ldots\}$,

(C7) for a compact set $\boldsymbol{S} \subseteq \boldsymbol{R}^{p}$ such that $\dot{\eta}(s)=-\nabla F(\eta(s))$ with $\eta(0) \in \boldsymbol{S}$ results in $\eta(\infty)=\zeta^{*}, \zeta(k) \in \boldsymbol{S}$ occurs infinitely often for almost all sample points of $\theta(k)(k=$ $0,1, \ldots)$,

hold, then

$$
\lim _{k \rightarrow \infty} \zeta(k)=\zeta^{*} \text { w.p.1. }
$$

Lemma 3 implies that, under several conditions, the system (A.1) converges to a local minimum point of the function $F$.

\section{B Proofs of Facts (a)-(c) in the Proof of Lemma 1}

\section{B1 Preliminary}

A preliminary result is provided.

Lemma 4: For the system $\Sigma$, suppose that $r \in \boldsymbol{R}_{+}$is given. Suppose also that $L_{i 1}(i=1,2, \ldots, N)$ are given by (8), (13), (14), and (15), $L_{i 2}(i=1,2, \ldots, N)$ are arbitrarily given so as to satisfy (A0), and $B$ is arbitrarily given. Then,

$$
\begin{aligned}
& \xi_{i 1}(t+N+2)=\xi_{i 1}(t+N+1) \\
& =\operatorname{sign}\left(\operatorname{abmax}\left(\left\{\Delta_{j}(t)\right\}_{j \in \mathbf{G}_{k}(t)}\right)\right)\left(\forall i \in \mathbf{G}_{k}(t)\right)
\end{aligned}
$$

holds for every $t \in \boldsymbol{T}_{1}$ and $k \in\{1,2, \ldots, M(t)\}$.

Proof: The first equality is trivial from (8), (13), and $t+N+1$ $\notin \mathbf{T}_{1} \cup \mathbf{T}_{2} \cup \mathbf{T}_{3}$ for $t \in \mathbf{T}_{1}$.

Meanwhile, the second equality is proved as follows. For $t \in$ $\mathbf{T}_{1}$, consider time $s \in\{t, t+1, \ldots, t+N\}$. Let $\xi_{i 11}(s)$ and $\Delta_{j 1}(s)$ be the first element of $\xi_{i 1}(s)$ and of $\Delta_{j}(s)$, respectively. Let us also divide the group $\mathbf{G}_{k}(s)$ into $\mathbf{G}_{k 1}(s):=\left\{i \in \mathbf{G}_{k}(t) \mid \xi_{i 11}(s)=\right.$ $\left.\operatorname{abmax}\left(\left\{\Delta_{j 1}(t)\right\}_{j \in \mathbf{G}_{k}(t)}\right)\right\}$ and $\mathbf{G}_{k 2}(s):=\mathbf{G}_{k}(s) \backslash \mathbf{G}_{k 1}(s)$. If $\mathbf{G}_{k 1}(t+$ $N)=\mathbf{G}_{k}(t)$ is proved, the 'first-row' part of the second equality holds for every $i \in \mathbf{G}_{k}(t)$ (since $\xi_{i 11}(t+N+1)=\operatorname{sign}\left(\xi_{i 11}(t+\right.$ $N)$ ) from (8) and (13)). On the other hand, it can be shown from (8), (13), (14), (15), (17), (A0), and the definition of the group that the following statements hold for $s \in\{t, t+1, \ldots, t+N$ $-1\}$ : (i) if $i \in \mathbf{G}_{k 1}(s)$, then $i \in \mathbf{G}_{k 1}(s+1)$ and (ii) if $\mathbf{G}_{k 2}(s) \neq \emptyset$ (i.e. $\left.\mathbf{G}_{k 1}(s) \neq \mathbf{G}_{k}(s)\right)$, there exists an $i \in \mathbf{G}_{k 2}(s)$ such that $i \in \mathbf{G}_{k 1}(s$ $+1)$. So, $\mathbf{G}_{k 2}(s) \neq \emptyset$ implies $\mathbf{G}_{k 1}(s) \subset \mathbf{G}_{k 1}(s+1)$, namely $\mathbf{G}_{k 1}(t$ $+N)=\mathbf{G}_{k}(t)$ due to $\left|\mathbf{G}_{k}(t)\right| \leq N$ and $\mathbf{G}_{k 1}(t) \neq \emptyset$. Therefore, the first-row part of the second equality holds for every $i \in \mathbf{G}_{k}(t)$. In a similar way, we can prove that the other-row parts hold for every $i \in \mathbf{G}_{k}(t)$. In this way, the second equality is obtained.

\section{B2 Proof of Fact (a)}

For $t \in \mathbf{T}_{1}(\tau)$, we have

$z(t+N+3)=z(t)-a(t) \frac{w(t+N+2)-w(t)}{c(t)} \Delta^{(-1)}(t)$,

$w(t)=J(x(t)), w(t+N+2)=J(x(t+N+2))$,
$J(x(t+N+2))-J(x(t))=J_{c}(z(t+N+2))-J_{c}(z(t))$,

$z(t+N+2)=z(t)+c(t) \Delta(t)$

subject to (A0) and (A1). Applying (B.3), (B.4), and (B.5) to (B.2) establishes (a).

In the following part, we prove (B.2), (B.3), (B.4), and (B.5)

\section{B2.1 Proof of (B.2)}

Consider $t \in \mathbf{T}_{1}(\tau)$. Equations (8), (13), and (17) imply

$$
\xi_{i 2}(t+N+2)=w(t) .
$$

In addition, (1), (8), (14), (A0), and Figure 5 provide

$$
\begin{aligned}
x_{i}(t+N+2) & =x_{i}(t)+u_{i}(t)+u_{i}(t+1)+\cdots+u_{i}(t+N+1) \\
& =x_{i}(t)+c(t+N+1) \xi_{i 1}(t+N+1) .
\end{aligned}
$$

Using these relations with (1), (8), (14), Figure 5, (A1), and Lemma 4, we obtain

$$
\begin{aligned}
x_{i}(t+N+3)= & x_{i}(t+N+2)+u_{i}(t+N+2) \\
= & x_{i}(t)+c(t+N+1) \xi_{i 1}(t+N+1) \\
& -c(t+N+2) \xi_{i 1}(t+N+2) \\
& -a(t+N+2) \frac{w(t+N+2)-\xi_{i 2}(t+N+2)}{c(t+N+2)} \\
& \times \xi_{i 1}^{(-1)}(t+N+2)+u_{i 2}(t+N+2) \\
= & x_{i}(t)-a(t) \frac{w(t+N+2)-w(t)}{c(t)} \\
& \times \xi_{i 1}^{(-1)}(t+N+2)+u_{i 2}(t+N+2) .
\end{aligned}
$$

Since the definition of $\tau$ and (11) imply that

$$
M(t)=M(\tau), \mathbf{G}_{k}(t)=\mathbf{G}_{k}(\tau)
$$

for every $t \in \mathbf{T}_{1}(\tau)$, (B.8) can be rewritten in the centroid form as

$$
\begin{aligned}
z_{k}(t & +N+3) \\
= & \frac{1}{\left|\mathbf{G}_{k}(\tau)\right|} \sum_{i \in \mathbf{G}_{k}(\tau)} x_{i}(t+N+3) \\
= & \frac{1}{\left|\mathbf{G}_{k}(\tau)\right|}\left(\sum_{i \in \mathbf{G}_{k}(\tau)} x_{i}(t)\right. \\
& -\sum_{i \in \mathbf{G}_{k}(\tau)} a(t) \frac{w(t+N+2)-w(t)}{c(t)} \xi_{i 1}^{(-1)}(t+N+2) \\
& \left.+\sum_{i \in \mathbf{G}_{k}(\tau)} u_{i 2}(t+N+2)\right) \\
= & z_{k}(t)-a(t) \frac{w(t+N+2)-w(t)}{c(t)} \\
& \times\left(\operatorname { s i g n } \left(\operatorname { a b m a x } \left(\left\{\Delta_{j}(t)\right\}_{\left.\left.\left.j \in \mathbf{G}_{k}(\tau)\right)\right)\right)^{(-1)}}\right.\right.\right.
\end{aligned}
$$


by (18), (A0), Lemma 4, and $t+N+2 \in \mathbf{T}_{5}$ for $t \in \mathbf{T}_{1}$. Note here that $\sum_{i \in \mathbf{G}_{k}(\tau)} u_{i 2}(t+N+2)=0$ due to (A0) and the relation $\mathbf{G}_{k}(\tau)=\mathbf{G}_{k}(t+N+2)$ which is given by the definition of $\tau$, (11), and (12). Putting (B.10) for $k=1,2, \ldots, M(\tau)$ together and using (21), we get (B.2).

\section{B2.2 Proof of (B.3)}

Trivial from (3) and (16).

\section{B2.3 Proof of (B.4)}

The performance index $J(x(t))$ in (6) is expressed as

$$
\begin{aligned}
J(x(t))= & \sum_{k=1}^{M(\tau)} \sum_{i \in \mathbf{G}_{k}(\tau)}\left\|x_{i}(t)-z_{k}(t)+z_{k}(t)-x_{d}\right\|^{2} \\
= & \sum_{k=1}^{M(\tau)} \sum_{i \in \mathbf{G}_{k}(\tau)}\left\|x_{i}(t)-z_{k}(t)\right\|^{2} \\
& +2 \sum_{k=1}^{M(\tau)} \sum_{i \in \mathbf{G}_{k}(\tau)}\left(x_{i}(t)-z_{k}(t)\right)^{\top}\left(z_{k}(t)-x_{d}\right) \\
& +\sum_{k=1}^{M(\tau)} \sum_{i \in \mathbf{G}_{k}(\tau)}\left\|z_{k}(t)-x_{d}\right\|^{2} .
\end{aligned}
$$

Then, since (8), (14), (27), (B.9), and (A0) imply

$$
u_{i 1}(s)=u_{j 1}(s), u_{i 2}(s)=u_{j 2}(s)\left(\forall(i, j) \in \mathbf{G}_{k}(\tau)\right)
$$

for $s \in\{t, t+1, \ldots, t+N+1\}$ and $t \in \mathbf{T}_{1}(\tau)$, it follows from (1), (18), and Figure 5 that

$$
\begin{aligned}
& x_{i}(s+1)-z_{k}(s+1) \\
&=x_{i}(s)+u_{i}(s)-\frac{1}{\left|\mathbf{G}_{k}(\tau)\right|} \sum_{j \in \mathbf{G}_{k}(\tau)}\left(x_{j}(s)+u_{j}(s)\right) \\
&=x_{i}(s)+u_{i 1}(s)+u_{i 2}(s) \\
& \quad-\frac{1}{\left|\mathbf{G}_{k}(\tau)\right|} \sum_{j \in \mathbf{G}_{k}(\tau)}\left(x_{j}(s)+u_{j 1}(s)+u_{j 2}(s)\right) \\
&=x_{i}(s)+u_{i 1}(s)+u_{i 2}(s)-u_{i 1}(s)-u_{i 2}(s) \\
&-\frac{1}{\left|\mathbf{G}_{k}(\tau)\right|} \sum_{j \in \mathbf{G}_{k}(\tau)} x_{j}(s) \\
&= x_{i}(s)-z_{k}(s)
\end{aligned}
$$

for $s \in\{t, t+1, \ldots, t+N+1\}$. Moreover,

$$
\sum_{i \in \mathbf{G}_{k}(\tau)}\left(x_{i}(t)-z_{k}(t)\right)^{\top}\left(z_{k}(t)-x_{d}\right)=0
$$

because $\sum_{i \in \mathbf{G}_{k}(\tau)}\left(x_{i}(t)-z_{k}(t)\right)^{\top}\left(z_{k}(t)-x_{d}\right)=\left(\sum_{i \in \mathbf{G}_{k}(\tau)} x_{i}(t)-\right.$ $\left.z_{k}(t)\right)^{\top}\left(z_{k}(t)-x_{d}\right)=\left(\left|\mathbf{G}_{k}(\tau)\right| z_{k}(t)-\left|\mathbf{G}_{k}(\tau)\right| z_{k}(t)\right)^{\top}\left(z_{k}(t)-x_{d}\right)=0$ from (18).
Applying (22), (B.11), (B.12), and (B.13) to $J(x(t+N+2))$ $-J(x(t))\left(\right.$ for $\left.t \in \mathbf{T}_{1}(\tau)\right)$, we obtain

$$
\begin{aligned}
& J(x(t+N+2))-J(x(t)) \\
& =\sum_{k=1}^{M(\tau)} \sum_{i \in \mathbf{G}_{k}(\tau)}\left\|x_{i}(t+N+2)-z_{k}(t+N+2)\right\|^{2} \\
& \quad+\sum_{k=1}^{M(\tau)}\left|\boldsymbol{G}_{k}(\tau)\right|\left\|z_{k}(t+N+2)-x_{d}\right\|^{2} \\
& \quad-\sum_{k=1}^{M(\tau)} \sum_{i \in \mathbf{G}_{k}(\tau)}\left\|x_{i}(t)-z_{k}(t)\right\|^{2} \\
& \quad-\sum_{k=1}^{M(\tau)}\left|\boldsymbol{G}_{k}(\tau)\right|\left\|z_{k}(t)-x_{d}\right\|^{2} \\
& =J_{c}(z(t+N+2))-J_{c}(z(t)) .
\end{aligned}
$$

In this equation, the first equality is derived by (B.11), (B.13), and the fact that $\left\|z_{k}(t+N+2)-x_{d}\right\|^{2}$ and $\left\|z_{k}(t)-x_{d}\right\|^{2}$ are independent of $i \in \mathbf{G}_{k}(\tau)$ and the second equality is given by (22) and (B.12) (note that (B.12) implies $\sum_{k=1}^{M(\tau)} \sum_{i \in \mathbf{G}_{k}(\tau)} \| x_{i}(t+N+$ $\left.2)-z_{k}(t+N+2)\left\|^{2}=\sum_{k=1}^{M(\tau)} \sum_{i \in \mathbf{G}_{k}(\tau)}\right\| x_{i}(t)-z_{k}(t) \|^{2}\right)$.

\section{B2.4 Proof of (B.5)}

Consider again $t \in \mathbf{T}_{1}(\tau)$. From (18), (B.7), (B.9), (A1), and Lemma 4 , the following relation holds:

$$
\begin{aligned}
z_{k}(t+N+2)= & \frac{1}{\left|\mathbf{G}_{k}(\tau)\right|} \sum_{i \in \mathbf{G}_{k}(\tau)} x_{i}(t+N+2) \\
= & \frac{1}{\left|\mathbf{G}_{k}(\tau)\right|} \sum_{i \in \mathbf{G}_{k}(\tau)}\left(x_{i}(t)+c(t+N+2)\right. \\
& \left.\times \xi_{i 1}(t+N+2)\right) \\
= & z_{k}(t)+\frac{c(t)}{\left|\mathbf{G}_{k}(\tau)\right|} \sum_{i \in \mathbf{G}_{k}(\tau)} \xi_{i 1}(t+N+2) \\
= & z_{k}(t)+c(t) \operatorname{sign}\left(\operatorname{abmax}\left(\left\{\Delta_{j}(t)\right\}_{j \in \mathbf{G}_{k}(\tau)}\right)\right.
\end{aligned}
$$

Thus, (B.5) is given by putting (B.14) for $k=1,2, \ldots, M(\tau)$ together.

\section{B3 Proof of Fact (b)}

We prove that conditions $(\mathrm{C} 1)-(\mathrm{C} 7)$ in Lemma 3 are satisfied for the dynamics in (20) subject to (A1) and (A2).

First, it is trivial that (22) and (A1) imply (C1)-(C3).

Next, from (21), (A2), and the definitions of 'sign' and 'abmax', it turns out that the random vectors $\Delta(t)(t=1,2$, ...) are independent of each other, and the elements of $\Delta(t)$ are equivalent to i.i.d. random variables from the Bernoulli trial with outcome \pm 1 and equal probabilities. So (C4) is satisfied.

Finally, (22) and Theorem 7 in Borkar (2008) give (C5). Moreover, (C6) and (C7) are proved by (C5) and (22) (in particular, the convexity of $J_{c}$ ).

\section{B4 Proof of Fact (c)}

It is trivial from (22). 


\section{Proof of Theorem 2 (i) and Facts (a)-(c) in the proof}

We show the proofs in order of Facts (a)-(c) and Statement (i).

\section{C1 Proofs of the three facts}

\section{C1.1 Proof of Fact (a)}

Suppose that $i \in\{0,1, \ldots, N\}$ and $t \in \mathbf{T}_{1}$ are arbitrarily given.

If $\mathbf{N}_{i}\langle h t\rangle=\emptyset$, then (34) obviously holds.

On the other hand, if $\mathbf{N}_{i}\langle h t\rangle \neq \emptyset$, (34) is proved in the following way. Consider the group $\mathbf{G}_{l}\langle h t\rangle$ satisfying $i \in \mathbf{G}_{l}\langle h t\rangle$. Let $x_{(l)}\left\langle t_{c}\right\rangle \in$ $\mathbf{R}^{n\left|\mathbf{G}_{l}\langle h t\rangle\right|}$ be the collective vector composed of $x_{j}\left\langle t_{c}\right\rangle$ for all $j \in$ $\mathbf{G}_{l}\langle h t\rangle$, and let

$$
V_{l}\left\langle t_{c}\right\rangle:=\sum_{k \in \mathbf{G}_{l}\langle h t\rangle} V_{k}\left(x_{k}\left\langle t_{c}\right\rangle,\left\{x_{j}\left\langle t_{c}\right\rangle\right\}_{j \in \mathbf{N}_{k}\langle h t\rangle}\right)
$$

for

$$
V_{k}\left(x_{k}\left\langle t_{c}\right\rangle,\left\{x_{j}\left\langle t_{c}\right\rangle\right\}_{j \in \mathbf{N}_{k}\langle h t\rangle}\right):=\sum_{j \in \mathbf{N}_{k}\langle h t\rangle} \frac{\left\|x_{j}\left\langle t_{c}\right\rangle-x_{k}\left\langle t_{c}\right\rangle\right\|^{2}}{r-\left\|x_{j}\left\langle t_{c}\right\rangle-x_{k}\left\langle t_{c}\right\rangle\right\|} .
$$

Then, it can be shown in the same way as Ji and Egerstedt (2007) (see also Appendix C.3) that if

$$
\frac{d V_{l}\left\langle t_{c}\right\rangle}{d t_{c}} \leq 0\left(\forall t_{c} \in[h t, h(t+N+3))\right),
$$

then

$$
\left\|x_{j}\left\langle t_{c}\right\rangle-x_{i}\left\langle t_{c}\right\rangle\right\|<r\left(\forall j \in \mathbf{N}_{i}\langle h t\rangle, \forall t_{c} \in[h t, h(t+N+3)]\right) .
$$

Therefore, if (C.17) holds, then $j \in \mathbf{N}_{i}\langle h t\rangle$ implies $j \in \mathbf{N}_{i}\left\langle t_{c}\right\rangle$ for every $t_{c} \in[h t, h(t+N+3)]$ (agent $j$ remains a neighbour of agent $i$ ), i.e. (34) holds.

Meanwhile, (C.17) is proved as follows. Assume $t_{c} \in[h t, h(t$ $+N+3)]$. In a similar way to defining the vector $x_{(l)}\left\langle t_{c}\right\rangle$, let $u_{(l)}\left\langle t_{c}\right\rangle, u_{(l) 1}\left\langle t_{c}\right\rangle$, and $u_{(l) 2}\left\langle t_{c}\right\rangle$ be the collective vectors of $u_{j}\left\langle t_{c}\right\rangle$, $u_{j 1}\left\langle t_{c}\right\rangle$, and $u_{j 2}\left\langle t_{c}\right\rangle$ for the group $\mathbf{G}_{l}\langle h t\rangle$. Since

$$
\frac{\partial V_{l}\left\langle t_{c}\right\rangle}{\partial x_{k}}=\sum_{j \in \mathbf{N}_{k}\langle h t\rangle} \frac{2 r-\left\|x_{j}\left\langle t_{c}\right\rangle-x_{k}\left\langle t_{c}\right\rangle\right\|}{\left(r-\left\|x_{j}\left\langle t_{c}\right\rangle-x_{k}\left\langle t_{c}\right\rangle\right\|\right)^{2}}\left(x_{k}\left\langle t_{c}\right\rangle-x_{j}\left\langle t_{c}\right\rangle\right)
$$

for $k \in \mathbf{G}_{l}\langle h t\rangle$, we obtain

$$
\begin{aligned}
u_{(l)}\left\langle t_{c}\right\rangle & =u_{(l) 1}\left\langle t_{c}\right\rangle+u_{(l) 2}\left\langle t_{c}\right\rangle \\
& = \begin{cases}\mathbf{1}_{\left|\mathbf{G}_{l}\langle h t\rangle\right|} u_{i 1}\left\langle t_{c}\right\rangle-\frac{\partial V_{l}\left\langle t_{c}\right\rangle}{\partial x_{(l)}} & \text { if } t_{c} \in \mathbf{T}_{5 c} \\
\mathbf{1}_{\left|\mathbf{G}_{l}\langle h t\rangle\right|} u_{i 1}\left\langle t_{c}\right\rangle & \text { otherwise }\end{cases}
\end{aligned}
$$

from Figure 5, (32) (in which $\mathbf{S}\left(\zeta_{i}\left\langle t_{c}\right\rangle\right)=\mathbf{N}_{i}\langle h t\rangle$ ), (C.15), (C.16), and the fact

(B0') for every $t \in \mathbf{T}_{1}$ and $k \in\{1,2, \ldots, M\langle h t\rangle\}$, $u_{i 1}\left\langle t_{c}\right\rangle=u_{j 1}\left\langle t_{c}\right\rangle$

$\left(\forall t_{c} \in[h t, h(t+N+3)), \forall(i, j) \in \mathbf{G}_{k}\langle h t\rangle \times \mathbf{G}_{k}\langle h t\rangle\right)$ for $L_{i 1}(i=1,2, \ldots, N)$. It follows from (29), (32), (C.19), and (C.20) that

$$
\begin{aligned}
& \frac{d V_{l}\left\langle t_{c}\right\rangle}{d t_{c}} \\
& =\left(\frac{\partial V_{l}\left\langle t_{c}\right\rangle}{\partial x_{(l)}}\right)^{\top} \dot{x}_{(l)}\left\langle t_{c}\right\rangle \\
& =\left\{\begin{array}{ll}
\left(\frac{\partial V_{l}\left\langle t_{c}\right\rangle}{\partial x_{(l)}}\right)^{\top}\left(\mathbf{1}_{\mid \mathbf{G}_{l}\langle h t\rangle} u_{i 1}\left\langle t_{c}\right\rangle-\frac{\partial V_{l}\left\langle t_{c}\right\rangle}{\partial x_{(l)}}\right) & \text { if } t_{c} \in \mathbf{T}_{5 c} \\
\left(\frac{\partial V_{l}\left\langle t_{c}\right\rangle}{\partial x_{(l)}}\right)^{\top} & \mathbf{1}_{\left|\mathbf{G}_{l}\langle h t\rangle\right|} u_{i 1}\left\langle t_{c}\right\rangle
\end{array}\right. \text { otherwise } \\
& = \begin{cases}-\left\|\frac{\partial V_{l}\left\langle t_{c}\right\rangle}{\partial x_{(l)}}\right\|^{2} & \text { if } t_{c} \in \mathbf{T}_{5 c} \\
0 & \text { otherwise }\end{cases} \\
& \leq 0 \quad 0
\end{aligned}
$$

for every $t_{c} \in[h t, h(t+N+3))$.

In this way, Fact (a) is proved.

\section{C1.2 Proof of Fact (b)}

In (32), $\mathbf{S}\left(\zeta_{i}\left\langle t_{c}\right\rangle\right)=\mathbf{N}_{i}\left\langle h t_{1}\right\rangle$ holds. This and the definition of the groups provide (b).

\section{C1.3 Proof of Fact (c)}

Let $\tau$ be a $t \in \mathbf{T}_{1}$ satisfying $s \leq t$ and (35). Since Fact (a) implies that $\mathbf{G}_{k}\langle h \tau\rangle=\mathbf{G}_{k}\langle h(\tau+N+3)\rangle=\mathbf{G}_{k}\langle h(\tau+(N+3) 2)\rangle=$ $\cdots=\mathbf{G}_{k}\langle\infty\rangle$ for every $k \in\{1,2, \ldots, M\langle h \tau\rangle\}$, it follows from (29) and (C.20) that the collective dynamics of the group $\mathbf{G}_{l}\langle h \tau\rangle$ is expressed as

$$
\dot{x}_{(l)}\left\langle t_{c}\right\rangle= \begin{cases}\mathbf{1}_{\left|\mathbf{G}_{l}\langle h t\rangle\right|} u_{i 1}\left\langle t_{c}\right\rangle-\frac{\partial V_{l}\left\langle t_{c}\right\rangle}{\partial x_{(l)}} & \text { if } t_{c} \in \mathbf{T}_{5 c} \\ \mathbf{1}_{\left|\mathbf{G}_{l}\langle h t\rangle\right|} u_{i 1}\left\langle t_{c}\right\rangle & \text { otherwise }\end{cases}
$$

for $t_{c} \in[h \tau, \infty)$. This corresponds to a consensus algorithm, because the input term $\mathbf{1}_{\mid \mathbf{G}_{l}\langle h t\rangle} u_{i 1}\left\langle t_{c}\right\rangle$ keeps the relative positions $x_{i}\left\langle t_{c}\right\rangle-x_{j}\left\langle t_{c}\right\rangle$ constant for every $(i, j) \in \mathbf{G}_{l}\langle h \tau\rangle \times \mathbf{G}_{l}\langle h \tau\rangle$, the input term $-\partial V_{i}\left\langle t_{c}\right\rangle / \partial x_{(l)}$ achieves the consensus as proved in $\mathrm{Ji}$ and Egerstedt (2007), and the supremum of $\mathbf{T}_{5 c}$ is infinity.

Meanwhile, in a similar way to the proof of Lemma 2, it turns out from $s \leq \tau$ and the definition of the groups that, for each $k \in$ $\{1,2, \ldots, \bar{M}\langle h s\rangle\}$, there exists an $l \in\{1,2, \ldots, M\langle h \tau\rangle\}$ such that $\mathbf{G}_{k}\langle h s\rangle \subseteq \mathbf{G}_{l}\langle h \tau\rangle$.

These complete the proof.

\section{C2 Proof of (i)}

In (32), $\mathbf{S}\left(\zeta_{i}\left\langle t_{c}\right\rangle\right)=\mathbf{N}_{i}\langle h t\rangle$ holds for every $t_{c} \in[h t, h(t+N+$ $3)$ ). This and Fact (a) imply (i).

\section{C3 Proof that (C.17) implies (C.18)}

Finally, we prove that (C.17) implies (C.18).

Let $\phi(d):=d^{2} /(r-d)$ for $d \in \mathbf{R} \backslash\{r\}$. From (28) and the definition of the groups, there exists an $\varepsilon \in \mathbf{R}_{+}$such that $\| x_{j}\langle h t\rangle$ $-x_{k}\langle h t\rangle \| \leq r-\varepsilon$ for every $(j, k) \in \mathbf{G}_{l}\langle h t\rangle \times \mathbf{G}_{l}\langle h t\rangle$. Moreover, $\phi$ is a monotonically increasing function with respect to $d \in$ 
1116

$[0, r)$. These and (C.17) imply

$$
\begin{aligned}
& \sum_{k \in \mathbf{G}_{l}\langle h t\rangle} \sum_{j \in \mathbf{N}_{k}\langle h t\rangle} \phi\left(\left\|x_{j}\left\langle t_{c}\right\rangle-x_{k}\left\langle t_{c}\right\rangle\right\|\right) \\
& \leq \sum_{k \in \mathbf{G}_{l}\langle h t\rangle} \sum_{j \in \mathbb{N}_{k}\langle h t\rangle} \phi(r-\varepsilon) \\
& =E \frac{(r-\varepsilon)^{2}}{\varepsilon}
\end{aligned}
$$

for every $t_{c} \in[h t, h(t+N+3))$, where $E:=\sum_{k \in \mathbf{G}_{l}\langle h t\rangle}\left|\mathbf{N}_{k}\langle h t\rangle\right|$. Here, $\phi\left(\left\|x_{j}\left\langle t_{c}\right\rangle-x_{k}\left\langle t_{c}\right\rangle\right\|\right) \geq 0$ for every $(j, k) \in \mathbf{G}_{l}\langle h t\rangle \times \mathbf{G}_{l}\langle h t\rangle$, and so (C.22) provides

$$
\phi\left(\left\|x_{j}\left\langle t_{c}\right\rangle-x_{i}\left\langle t_{c}\right\rangle\right\|\right) \leq E \frac{(r-\varepsilon)^{2}}{\varepsilon}
$$

for every $j \in \mathbf{N}_{i}\left\langle t_{c}\right\rangle$. Meanwhile,

$$
\phi\left(r-\frac{\varepsilon}{E}\right)=E \frac{\left(r-\frac{\varepsilon}{E}\right)^{2}}{\varepsilon} .
$$

Since $E \geq 1$ (under $\mathbf{N}_{i}\langle h t\rangle \neq \emptyset$ ) and $\phi$ is monotonically increasing, (C.23) and (C.24) establish

$$
\left\|x_{j}\left\langle t_{c}\right\rangle-x_{i}\left\langle t_{c}\right\rangle\right\| \leq r-\frac{\varepsilon}{E}
$$

for every $t_{c} \in[h t, h(t+N+3))$. This and (29) prove (C.18). 